TI 2012-018/4

Tinbergen Institute Discussion Paper
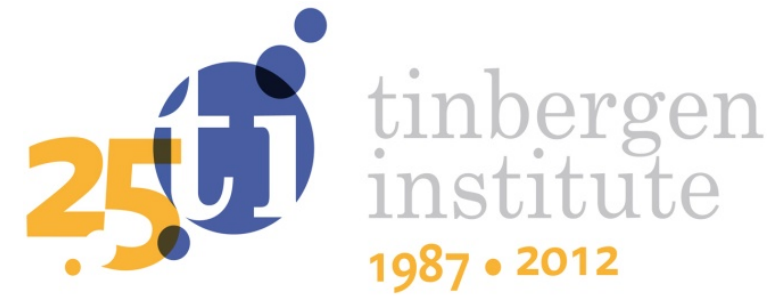

\title{
High-Frequency Technical Trading: The Importance of Speed
}

\author{
Martin L. Scholtus \\ Dick van Dijk
}

Econometric Institute, Erasmus School of Economics, Erasmus University Rotterdam, and Tinbergen Institute. 
Tinbergen Institute is the graduate school and research institute in economics of Erasmus University Rotterdam, the University of Amsterdam and VU University Amsterdam.

More TI discussion papers can be downloaded at http://www.tinbergen.nl

Tinbergen Institute has two locations:

Tinbergen Institute Amsterdam

Gustav Mahlerplein 117

1082 MS Amsterdam

The Netherlands

Tel.: +31(0)205251600

Tinbergen Institute Rotterdam

Burg. Oudlaan 50

3062 PA Rotterdam

The Netherlands

Tel.: +31(0)10 4088900

Fax: $+31(0) 104089031$

Duisenberg school of finance is a collaboration of the Dutch financial sector and universities, with the ambition to support innovative research and offer top quality academic education in core areas of finance.

DSF research papers can be downloaded at: http://www.dsf.nl/

Duisenberg school of finance

Gustav Mahlerplein 117

1082 MS Amsterdam

The Netherlands

Tel.: +31(0)20 5258579 


\title{
High-Frequency Technical Trading: The Importance of Speed
}

\author{
Martin L. Scholtus*, Dick van Dijk \\ Econometric Institute and Tinbergen Institute, Erasmus University Rotterdam \\ P.O. Box 1738, 3000 DR Rotterdam, The Netherlands
}

February 2012

\begin{abstract}
This paper investigates the importance of speed for technical trading rule performance for three highly liquid ETFs listed on NASDAQ over the period January 6, 2009 up to September 30, 2009. In addition we examine the characteristics of market activity over the day and within subperiods corresponding to hours, minutes, and seconds. Speed has a clear impact on the return of technical trading rules. For strategies that yield a positive return when they experience no delay, a delay of 200 milliseconds is enough to lower performance significantly. On low volatility days this is already the case for delays larger than 50 milliseconds. In addition, the importance of speed for trading rule performance increases over time. Market activity follows a U-shape over the day with a spike at 10:00AM due to macroeconomic announcements and is characterized by periodic activity within the day, hour, minute, and second.
\end{abstract}

Keywords: Technical Trading, High-Frequency Trading, Latency Costs, Trading Speed, Market Activity

JEL: G10, G14, G20

${ }^{*}$ Corresponding author.

Email addresses: scholtus@ese.eur.nl (Martin L. Scholtus), djvandijk@ese.eur.nl (Dick van Dijk) 


\section{Introduction}

In recent years financial markets have changed considerably due to technological progress. How important technology has become for the securities and investment industry can be observed from the investments in information technology, which in the US and Canada, for example, is estimated to have increased from $\$ 26$ billion ${ }^{1}$ in 2006 to $\$ 56$ billion ${ }^{2}$ in 2011 . One of the major technological developments is the appearance of new electronic trading platforms that offer low-cost, high-speed market access. A good example of such an exchange is Chi-X, which after its introduction in 2007 managed to become the second largest European exchange in terms of equity trading turnover by January 2010 $\mathrm{H}^{3}$ In the US Direct Edge and BATS together account for about $24 \%$ of all equity trading volume by September 2011.4 The most fundamental change, however, is the rise of automatic trading, which has become very dominant in developed markets in recent years. For example, during the years 2008 and $200968.5 \%$ of the (NASDAQ) trading volume can be attributed to high-frequency proprietary trading (Brogaard, 2010) $!^{5}$

The increase in high-frequency trading has many side effects, such as the phenomenon of flash crashes as occurred on May $6^{\text {th }} 2010$. This event put

\footnotetext{
${ }^{1}$ Finance And Economics: Moving markets; Technology and exchanges, The Economist, February 4, 2006.

2 http: //www . celent.com/reports/it-spending-banking-north-american-perspective-0

${ }^{3}$ Chi-X becomes second-largest European bourse, Financial Times, February 10, 2010.

${ }^{4}$ http://batstrading.com and https://www.directedge.com

${ }^{5}$ We follow Hendershott and Riordan (2011) by defining algorithmic trading as the use of computer algorithms to automatically make trading decisions, submit orders, and manage those orders after submissions. As in Brogaard (2010) and Gomber, Arndt, Lutat and Uhle (2011) high-frequency trading is considered to be a subset of algorithmic trading due to, for example, its proprietary nature, the large number of order submissions and cancellations and the focus on highly liquid instruments.
} 
high-frequency traders in the spotlight of both media and regulators and directly led to new regulation such as the introduction of individual stock circuit breakers ${ }^{6}$ and requirements for broker-dealers before providing their customers with direct access to an exchange.7 Despite the negative media attention Chaboud, Chiquoine, Hjalmarsson and Vega (2009), Brogaard (2010), Hendershott, Jones and Menkveld (2011), and Hendershott and Riordan (2011) find that algorithmic trading has a positive influence on market quality measures such as volatility, liquidity and the price discovery process. Furthermore, Kirilenko, Kyle, Samadi and Tuzun (2011) conclude that the flash crash on May 6, 2010 was not triggered by high-frequency traders. Instead, high-frequency traders intensified the downward price move initiated by fundamental sellers.

Algorithmic trading also leads to the dilution of traditional market microstructure concepts and, at the same time, to the introduction of new concepts. For example, Hasbrouck and Saar (2009) introduce the notion of fleeting orders, which are nonmarketable limit orders that are cancelled within a short time interval after having been submitted to the exchange. Hasbrouck and Saar (2009) document that, for a sample of 100 NASDAQ listed stocks traded on INET during October 2004, 36.69\% (11.5\%) of the limit orders are fleeting and get cancelled within 2 seconds (100 milliseconds). On the other hand, O'Hara (2010) describes how fleeting orders (but also, for example, flash orders or 'match only' orders) lead to a situation where it is no longer clear what can be considered a quote and argues for a re-examination

\footnotetext{
${ }^{6}$ http://www.sec.gov/news/press/2010/2010-98.htm

7 http://www.sec.gov/news/press/2010/2010-210.htm
} 
of this basic concept.

The new electronic trading platforms and large investments in technology suggest that speed is more and more becoming a key for success in financial markets. However, to the best of our knowledge, no studies exist that quantify the cost of being slow for actual trading strategies. The main contribution of this paper is an analysis of the impact of speed on basic technical trading strategy performance with millisecond precision. We use three highly liquid Exchange Traded Funds (ETFs) traded on NASDAQ over the period January 6, 2009 up to September 30, 2009 8 The ETFs used in the analysis are SPY (S\&P 500), QQQQ (NASDAQ 100), and IWM (Russell 2000). We apply 27,424 technical trading rules, comparing their performance when buy and sell orders are executed instantaneously with their performance when execution is delayed by a small amount of time. We find that delays have a negative impact on trading rule profitability. The decline in performance is significant when the delay is larger than 200 milliseconds (ms). On days with low volatility this is already the case for delays of $50 \mathrm{~ms}$ and up. A regression of the importance of speed on a time trend suggests that speed becomes more important over time. All results are robust with respect to the choice of the ETF, the capacity level of the trade, and the interval choice of the technical trader. The analysis can directly aid practitioners in decision making regarding investments in information technology by investors.

In addition to the importance of speed, we investigate the high-frequency

\footnotetext{
${ }^{8}$ Note that this is the first research on market activity of highly liquid ETFs during a longer time period. It is therefore unlikely that specific events during a particular week or month influence the results.
} 
trading environment by looking at patterns in message activity during the day, hour, minute, and second 9 The observed intraday regularities consist of a U-shape of message activity over the day as well as patterns over the hour and minute that suggest periodic activities of high-frequency traders. The oscillations within the second reveal traders that, due to differences in geographic location and technology, act with different speed levels.

Trading on regular, clock based, intervals is part of many simple technical trading rules that try to predict future price moves of securities based on historical price and volume data as inputs. These practices are not new to financial markets and are already documented by, for example, Fama and Blume (1966), Brock, Lakonishok and LeBaron (1992), and Gençay (1998). Technical analysis is widely used in practice by, for example, foreign exchange professionals (Menkhoff and Taylor, 2007) and equity fund managers (Menkhoff, 2010). The findings with respect to the profitability of technical trading vary widely and depend on the type of market and time period of the analysis as well as choices with respect to the data frequency, the treatment of transaction costs, and possible checks for datasnooping. For example, for equity markets see Hsu and Kuan (2005) and Marshall, Cahan and Cahan (2008) ${ }^{10}$ Recently, Schulmeister (2009) shows that the profitability of technical trading has shifted from daily to intraday data.

Little work has been done to analyse the importance of speed on trading

\footnotetext{
${ }^{9}$ Message activity entails every order market participants submit to the exchange.

${ }^{10}$ For commodities compare the results of Marshall, Cahan and Cahan (2008) and Szakmary, Shen and Sharma (2010). For currency markets see Neely, Weller and Dittmar (1997), Olson (2004), Qi and Wu (2006), or Cialenco and Protopapadakis (2011). A review is provided by Park and Irwin (2007).
} 
performance. Moallemi and Saglam (2010) introduce a theoretical model resulting in a cost of latency that depends on speed and the volatility and bidask spread of the asset. Ende, Uhle and Weber (2011) provide an empirical analysis using data of six DAX30 stocks traded on Deutsche Boerse over the period August 31, 2009 through September 11, 2009. The relative frequency of adverse orderbook changes and the corresponding average magnitude of the change are used to construct an estimate of the cost of latency for submitting market orders without considering specific trading strategies 11 The results show that the probability of unfavourable orderbook changes increases with latency and market capitalization. An overall estimate of the cost of latency is not provided ${ }^{12}$

There is a wide variety of intraday patterns in financial markets, ranging from U-shaped bid-ask spreads and trading volumes over the day (see, for example, McInish and Wood (1992) and Lehmann and Modest (1994)) to patterns in the quality of order execution (Garvey and $\mathrm{Wu}, 2009$ ). The regularities of message intensity and trader activity described in this paper relate to the work of Hasbrouck and Saar (2011) and Prix, Loistl and Huetl (2007). In Hasbrouck and Saar (2011) two types of high-frequency traders are identified. The first type of trader responds to market events (such as changes in the orderbook), whereas the second type acts according to a fixed time schedule. Peaks in activity within the second are contributed to traders that

\footnotetext{
${ }^{11}$ Ende et al. (2011) also try to find the cost of latency for submitting limit orders, however, without a model to price unfavourable volume changes in the orderbook or applying actual trading strategies this is not possible.

${ }^{12} \mathrm{An}$ estimate of the average cost of latency of 0.0282 basispoints (of the stock price) is only provided for market buy orders with a latency of $50 \mathrm{~ms}$.
} 
trade, due to their geographic location, with the same speed. Prix, Loistl and Huetl (2007) find evidence of periodic activity at a frequency of one minute for the individual DAX30 stocks during the periods from December 8 to December 15, 2004 and January 5 to January 12, 2005.

The outline of this paper is as follows. Section 2 provides a description of the NASDAQ ETF data used as well as an overview of intraday patterns in market activity for these assets. The details of technical trading rules and assumptions with respect to trade execution are discussed in Section 3. Results are presented in Section 4, followed by a robustness analysis and concluding remarks in Sections 5 and 6, respectively.

\section{Data}

\subsection{Data}

The dataset consists of full orderbook information for three highly liquid ETFs traded on the NASDAQ over the period January 6, 2009 up to September 30, 2009 (186 trading days) ${ }^{13}$ The three ETFs under consideration are SPY (State Street S\&P 500 ETF, the most liquid instrument traded on NASDAQ), QQQQ (Powershares NASDAQ 100 ETF), and IWM (iShares Russell 2000 ETF). NASDAQ does not directly provide the full orderbook, but this is constructed by means of the daily NASDAQ TotalView ITCH V4.0 files.

\footnotetext{
${ }^{13}$ The sample period is turbulent with a $-27 \%$ return on the S\&P500 from January up to the beginning of March. The period March through September is characterized with recovery leading to a year to date return of $22 \%$ at the end of September. There are no noteworthy individual events during the sample period.
} 
The TotalView ITCH files contain recordings of a direct data feed product offered by NASDAQ. The data elements in the file are order level data as well as trade messages, administrative messages, and net order imbalance data. We make use of order level data and administrative messages. The order level data consists of messages to add a new order (type A) and messages to modify existing orders (types E, C, X, D, and U) 14 The administrative messages provide system event messages (including, for example, start and end of system and market hours), information about trading halts and market participant positions. All messages have a timestamp with potential nanosecond precision. However, for this sample period the actual precision provided by NASDAQ is one million nanoseconds or one millisecond (ms).

There are two challenges in the construction of the orderbook. The first challenge is the large amount of data that must be processed. Both the input data and the output of the orderbook construction take almost two terabytes of storage space. The second challenge is the large amount of search operations resulting from the fact that only add order messages have a field with the stock ticker symbol. Once an order has been added to the orderbook it is referred to by means of the order identification number. Hence, for every message that does not add a new order the only way to determine whether the message (which can belong to any stock or ETF traded on NASDAQ) is relevant to the orderbook of a particular stock or ETF is by checking whether the order number is already in the orderbook. The construction

\footnotetext{
${ }^{14}$ Message type $\mathrm{E}$ and $\mathrm{C}$ are execution messages (where $\mathrm{E}$ is an execution against the price of the limit order originally put in and $\mathrm{C}$ is execution against a different price), whereas type $\mathrm{X}, \mathrm{D}$, and $\mathrm{U}$ are order cancellations, deletions, and replace messages, respectively.
} 
of the orderbook itself is a straightforward bookkeeping exercise that can be checked easily because the book, which starts empty at the beginning of system hours, must be empty again at the end of the day.

Note that from June 5, 2009 to August 31, 2009 it was possible to use flash orders on NASDAQ. The flash order functionality makes it possible that if an order has sweeped the top of the NASDAQ book, the remainder of the order is flashed on top of the book for a maximum of 500 ms instead of being routed to another exchange. If, after $500 \mathrm{~ms}$, the order is still marketable at another exchange, the order is cancelled. When the order is non-marketable it remains in the orderbook until cancelled by the customer. Flash orders are included in the analysis. Any results that are potentially affected by the inclusion of flash orders are tested for robustness.

\subsection{Intraday Patterns}

In Table 1 we provide summary statistics regarding the number of messages per type per day for each ETF during the complete sample period. Note that the order replace message (type U) is only supported by NASDAQ from the beginning of April, 2009 ${ }^{15}$ For the individual message types in $\mathrm{Ta}-$ ble 1 the statistics are calculated on a daily basis and then averaged across all days the message type is supported by NASDAQ. Because this number of days is less for $\mathrm{U}$ (which is available on 122 of the 186 trading days) the means of the individual message types do not exactly sum to the column TOTAL.

From Table 1 it is clear that SPY is the most liquid ETF in the sample,

\footnotetext{
${ }^{15}$ http://www . nasdaqtrader . com/TraderNews . aspx?id=dn2009-010
} 
with almost 5.7 million messages per day. The gap between SPY and IWM (about 3.9 million messages per day) and QQQQ (3.4 million messages) is substantial. Given a 6.5 hour trading day from 09:30AM to 04:00PM, the average number of messages per second is 243.5, 145.9, and 167.2 for SPY, QQQQ, and IWM, respectively. Most of the message activity consists of add order (A) and delete order (D) messages, which take up about $82 \%$ of the total message activity for SPY and QQQQ and $87.0 \%$ for IWM). ${ }^{16}$ The percentage of messages that are actually executions (of type $\mathrm{C}, \mathrm{E}$, or $\mathrm{P}$ ) is about $5.8 \%$ for SPY, 2.7\% for QQQQ, and 2.1\% for IWM. Because SPY has the highest absolute activity this will be the point of focus of further analysis in this section.

Next we investigate how the message intensity is distributed over the day, hour, minute, and second. The analysis over the day is performed by counting the number of messages during intervals of one minute. In order to obtain the message activity over the hour, minute, and second we consider intervals of one minute, one second and one millisecond, respectively. For the message activity within the hour, minute, and second we exclude the first 90 minutes and last 60 minutes of the trading day, to avoid unduly influence of these specific trading periods (which show by far the highest message activity).

Figure 1 shows the average message activity during the complete trading day. It appears that total message activity follows a U-shape, which is also found for intraday bid-ask spreads and trading volumes (McInish and Wood

\footnotetext{
${ }^{16}$ The percentage of $\mathrm{A}, \mathrm{D}$, and $\mathrm{E}$ messages during the flash order period is $41.35 \%$, $36.87 \%$, and $6.06 \%$, respectively, compared to $43.86 \%, 40.00 \%$, and $5.03 \%$ during days when flash trading is not possible. Hence, there is no substantial impact of flash trading.
} 
(1992) and Lehmann and Modest (1994)). At 10:00AM a large peak occurs in message intensity, which is a consequence of macroeconomic announcements. ${ }^{17}$ In addition, Figure 1 shows small peaks at full and half hours.

The average message activity during the hour and minute are displayed in Figure 2 and Figure 3, respectively. Within the hour we find peaks in message intensity just after the hour change, at the half hour change, and regular interval changes (for example the $5^{\text {th }}, 15^{\text {th }}$, and $45^{\text {th }}$ minute) 18 The message intensity per second within the minute, presented in Figure 3 , reveals a peak in intensity when the minute changes, whereas during the rest of the minute small peaks occur that do not match a regular pattern. The regularities during the hour and at the beginning of every minute can be explained by market participants that trade at a regular frequency.

Figure 4 provides the message intensity within the second. There is a large peak at $5 \mathrm{~ms}$ in the second and smaller peaks around $50 \mathrm{~ms}$ and 150 ms. Double peaks occur at approximately $250 \mathrm{~ms}, 500 \mathrm{~ms}$, and $750 \mathrm{~ms}$. Although more peaked, Figure 4 is quite similar to the findings of Hasbrouck and Saar (2011) for the year 2008 for individual stocks. As suggested by Hasbrouck and Saar (2011) the spikes can be caused by periodic traders with similar speed in the same geographic location that are triggered to trade by an interval change 19 The first peak at $5 \mathrm{~ms}$ is most likely due to co-located

\footnotetext{
${ }^{17}$ During the sample period we identify 91 days with macroeconomic announcements at 10:00AM. When Figure 1 is constructed excluding these days the spike disappears.

${ }^{18}$ Note that, for example, the peak at minute 16 is an average of the number of messages that arrives during the $16^{\text {th }}$ minute (starting at hh:15:00 up to (but not including) hh:16:00)

${ }^{19}$ It is likely that an algorithmic trader that is dedicated to sending large orders to the market (by using, for example, a VWAP algorithm) accesses the market at smaller frequencies, such as every second, whereas proprietary algorithms use a lower frequency
} 
servers, whereas the double peaks at $250 \mathrm{~ms}$, $500 \mathrm{~ms}$, and $750 \mathrm{~ms}$ are most likely caused by traders in the same geographic location, but with differences in hardware and complexity of trading algorithms (the more complex an algorithm the more time it takes to receive a trade signal). The pattern in Figure 4 does not change when the first five seconds of each minute (which are characterized by most activity) are excluded. The (unreported) graphs for IWM and QQQQ, corresponding to Figure 1 to Figure 4 of SPY, lead to the same conclusions.

\subsection{Fleeting Orders and Missed Opportunities}

In order to emphasize the importance of speed Figure 5(a) provides the percentage of fleeting orders with respect to the total number of limit orders added to the orderbook per ETF. The percentage of fleeting orders is determined for seven definitions of a fleeting order (nonmarketable limit orders added to and deleted from the orderbook within 10, 20, 50, 100, 200, and 500 ms, and 1 second). While about $15 \%$ of all message activity is fleeting within $10 \mathrm{~ms}$, this is already at least 30\% within $100 \mathrm{~ms}$, increasing further to $55 \%$ after 1 second. This is much higher than the 11.5\% reported by Hasbrouck and Saar (2009) for fleeting orders at the $100 \mathrm{~ms}$ level. An overview of the locations where fleeting orders (at the $50 \mathrm{~ms}$ level) enter the orderbook is presented in Figure 5(b). For all ETFs most (40\% in case of QQQQ and about $30 \%$ in case of SPY and IWM) fleeting orders are added at the current

best bid or ask (location 0). Furthermore, for all ETFs the percentage of fleeting orders added more than 5 cents from the best bid or ask is negligi-

to save upon transaction costs. 
ble. The percentage of fleeting orders that actually improves the best bid or ask is also close to zero. This is due to the fact that the overall number of improvements to the best bid or ask is low compared to total message activity. 20

In case of add order messages that improve the best bid or ask quote not only cancelled messages are important, but also the number of these messages that are fully executed. In Figure 5(c) we present the percentage of all improvements to the best bid or ask that are either cancelled or fully executed. Within $10 \mathrm{~ms} 24 \%$ (IWM) to $38 \%$ (SPY) of all improvements to the best bid or ask are either cancelled or fully executed. After 1 second this is $85 \%$ for SPY, 70\% for IWM, and 65\% for QQQQ. Figure 5(d) provides the number of missed opportunities, which is defined as the percentage of orders that improve the best bid or ask which are quickly removed or fully executed, leaving a worse book for the rest of the market. The percentage of missed opportunities is about $45 \%$ at a $10 \mathrm{~ms}$ interval and decreases to about $22 \%$ at the 1 second level. This can be explained by the fact that the longer a new order that improved the best bid or ask is in the book, the more time there is for other market participants to also submit orders at the new best bid or ask 21

The percentage of fleeting orders (at the $500 \mathrm{~ms}$ and 1 second level) and percentage of best bid-ask improvements and missed opportunities could be

\footnotetext{
${ }^{20}$ This is because most of the times the bid-ask spread is 1 cent leaving no additional room to improve upon.

${ }^{21}$ Note that the total impact on trading performance cannot be inferred from these results because the missed opportunities cannot be matched to trade signals (and the sign of the trade signal).
} 
influenced by the NASDAQ flash order functionality. Therefore, we also construct Figure 5 for the period with and without flash orders, which are provided in the internet appendix. It appears that the percentage improvements to the best bid or ask quote that dissapear (due to either cancellations or executions) is robust to the introduction of flash orders. The number of fleeting orders at the $500 \mathrm{~ms}$ and 1 second level, however, increases during the flash order period. For example, for SPY the percentage of fleeting orders at the $500 \mathrm{~ms}$ and 1 second level is about $42 \%$ and $52 \%$ (49\% and 59\%) for the period excluding (including) flash functionality. The percentage of missed opportunities increased during the period with flash orders for delay levels up to $200 \mathrm{~ms}$ (for example, for SPY, the percentage of missed opportunities at the $10 \mathrm{~ms}$ (200 ms) delay level is about $43 \%$ (28\%) for the period excluding flash orders, whereas this is about 50\% (31\%) for the period including flash orders).

Overall, the results in this section indicate that speed can be an important factor in the profitability of technical trading strategies. Not only does the fastest group of market participants react in $5 \mathrm{~ms}$ (see Figure 4), the percentage of improvements to the best bid or ask quote that dissapear within $10 \mathrm{~ms}$ (for SPY) is almost $40 \%$, of which almost $50 \%$ results in a worse orderbook for slower traders.

\section{Methodology}

\subsection{Trading Procedures}

All trading rules considered in this paper are 'periodic', meaning that trade signals are constructed throughout the day at a fixed, regular frequency 
based on the information available at that point in time. In our baseline analysis, we consider trading rules that construct a new signal every 60 seconds (i.e. at a one minute frequency). We define latency as the time it takes from the moment an order is sent to the exchange until it is processed, whereas speed is defined as the total time necessary to receive the latest market information at the interval change, construct a trading signal, and the latency resulting from sending an order to the exchange. This means that speed includes not only all potential sources of latency, such as location and network quality, it also includes other sources of delay, such as computational time to construct a trading signal. The impact of speed is investigated by introducing lags between the moment the information necessary to construct a signal is available and the moment a trade is actually executed. For example, in the case of no lag (or delay) a trade signal is acted upon instantaneously. In case of a delay of $20 \mathrm{~ms}$ the signal as calculated at a certain point during the day is executed $20 \mathrm{~ms}$ later. Eight different speed levels are considered: 0 (instantaneous), 10, 20, 50, 100, 200, and $500 \mathrm{~ms}$, and 1 second.

With the pre-market trading hours (ranging from 07:00:00 to 09:30:00) it is possible to have trading signals available immediately at the start of regular trading hours. However, to prevent distortions in the measurements concerning the importance of speed trading signals due to high activity around the opening (before 09:40:00) and closing times (after 15:50:00) are not acted upon. At 15:50:00 all open positions are unwinded. Because the focus of this paper is not on absolute profitability transaction costs, except for the bid-ask spread, are ignored.

The importance of speed is initially investigated for a buy/sell order in- 
volving 100 ETFs, whereas robustness checks are performed for a trade capacity of 500, 1,000, and 5,000 ETFs. For each capacity level a price-impact price per ETF is calculated as the average price paid for the capacity level number of ETFs. Hence, we account for the fact that a trade walks up the orderbook.

Because trade capacity is defined in number of ETFs, the dollar amounts invested differ for each trade (since the price changes). In order to calculate total returns, we assume a $\$ 1$ dollar investment in each strategy for each capacity level. In this way it is possible to calculate the total performance of a strategy as the sum of the simple percentage returns of each roundtrip trade. Returns are not compounded, because capacity is constant (you do not reinvest returns). The returns of buy $\left(r_{b u y, c, t 1, t 2}\right)$ and sell $\left(r_{\text {sell,c,t1,t2 }}\right)$ signals of a trade starting at time $t_{1}$ (the moment you take a position) to time $t_{2}$ (the moment a position is unwinded) for capacity level $c$ are calculated as follows:

$$
\begin{gathered}
r_{b u y, c, t 1, t 2}=\frac{p_{b i d, c, t 2}-p_{a s k, c, t 1}}{p_{a s k, c, t 1}} \\
r_{s e l l, c, t 1, t 2}=-1 \times \frac{p_{a s k, c, t 2}-p_{b i d, c, t 1}}{p_{b i d, c, t 1}}
\end{gathered}
$$

where $p_{b i d, c, t}\left(p_{a s k, c, t}\right)$ is the price-impact bid (ask) price at time $t$ for capacity level $c$.

\subsection{Trading Rules}

We consider seven different types of trading rules adopted from Sullivan, Timmermann and White (1999), Hsu and Kuan (2005), and Marshall et al. (2008). The Moving Average (MA), Filter (FI), Support and Resistance (SR), Channel Break-outs (CB), and Momentum in Price (MP) strategies 
are all price based. The remaining two trading rules, On Balance Volume Averages (OBV) and Momentum in Volume (MV), are volume based. All strategies, except for the FI rules, make use of lookback periods. The parameters $l$ and $s$ provide the length of the 'long' and 'short' lookback period in number of intervals with $l>s$. The main input for the trading rules is either the midpoint at time $t, m_{t}=\frac{p_{b i d, t}+p_{a s k, t}}{2}$, or $v_{t}$, the trading volume in terms of number of ETFs over the interval $t-1$ to $t$. Almost all strategies make use of a bandwith parameter $b$ to separate weak signals from stronger signals by increasing the threshold to be breached before a signal is provided. In the end all strategies lead to a raw trading signal at time $t, S_{t}$ with $S \in\{M A, \ldots, M V\} . S_{t}$ takes the values $1,-1$ or 0 , corresponding with a long, short and neutral position, respectively.

The signals for the trading rules are generated by means of the following three operators: $m a_{t}(l, x)=\frac{1}{l} \sum_{t=t-l+1}^{t} x_{t}, \operatorname{ret}_{t}(x)=\frac{x_{t}-x_{t-1}}{x_{t-1}}$, and $\operatorname{mar}_{t}(l, x)=m a_{t}(l, \operatorname{ret}(x))$. The first operator is a simple moving average whereas the other two operators represent a return and moving average of returns, respectively. The operators can be applied to either midpoints, with $x=m$, or volumes, with $x=v$.

For the MA, MP, and MV rules in Equation (3) to (5) holds that a long (short) position is entered when a recent average of an 'oscillator' (either the midpoint, return on midpoints, or return on volumes) is larger (smaller) than the average over a longer lookback period, indicating an increasing (decreasing) trend in the oscillator. Taking into account the bandwidth $b$, 
this can be formalized in the trading rules:

$$
\begin{gathered}
M A_{t}= \begin{cases}1 & \text { if } \operatorname{ma}_{t}(s, m)>(1+b) \cdot \operatorname{ma}_{t}(l, m) \\
-1 & \text { if } m a_{t}(s, m)<(1-b) \cdot m a_{t}(l, m) \\
0 & \text { otherwise }\end{cases} \\
M P_{t}= \begin{cases}1 & \text { if } \operatorname{mar}_{t}(s, m)>(1+b) \cdot \operatorname{mar}_{t}(l, m) \\
-1 & \text { if } \operatorname{mar}_{t}(s, m)<(1-b) \cdot \operatorname{mar}_{t}(l, m) \\
0 & \text { otherwise }\end{cases} \\
M V_{t}= \begin{cases}1 & \text { if } \operatorname{mar}_{t}(s, v)>(1+b) \cdot \operatorname{mar}_{t}(l, v) \\
-1 & \text { if } \operatorname{mar}_{t}(s, v)<(1-b) \cdot \operatorname{mar}_{t}(l, v) \\
0 & \text { otherwise }\end{cases}
\end{gathered}
$$

The OBV rule, provided in Equation (6) also makes use of the $m a_{t}$ operator as well as individual transaction data where trades are indexed from the first trade of the day $t r_{1}$ with price $p_{t r_{1}}$ to the last trade up to time $t$, denoted $t r_{t}$ with price $p_{t r_{t}}$. The obv operator is then defined as: $o b v_{t}=\sum_{j=t r_{1}}^{t r_{t}}\left(I\left(p_{j-1}>p_{j}\right) \cdot v_{j}-I\left(p_{j-1}<p_{j}\right) \cdot v_{j}\right)$, where $I(x)$ is an indicator function with value 1 if $x$ is true and 0 otherwise. Equation (6) results in a buy (sell) signal when the running count of signed trading volume has an 
increasing (decreasing) trend.

$$
O B V_{t}= \begin{cases}1 & \text { if } m a_{t}(s, o b v)>(1+b) \cdot m a_{t}(l, o b v) \\ -1 & \text { if } m a_{t}(s, o b v)<(1-b) \cdot m a_{t}(l, o b v) \\ 0 & \text { otherwise }\end{cases}
$$

For the SR rules we need the maximum (minimum) midpoint value over a lookback period of $l$ intervals, accounting for intra-interval maxima (minima). The maximum (minimum) during the interval from time $t-1$ to $t$ is denoted as $m_{t}^{\max }\left(m_{t}^{\min }\right)$, whereas the maximum (minimum) midpoint over a lookback period of $l$ intervals, $l b \max x_{t}$ is defined as: $\operatorname{lbmax}_{t}(l, m)=$ $\max \left(m_{t-1}^{\max } \ldots m_{t-1}^{\max }\right)$. When the support $\left(\operatorname{lbmin}_{t}\right)$ or resistance $\left(\operatorname{lbmax}_{t}\right)$ is breached by the current midpoint a sell or buy signal is provided, respectively.

$$
S R_{t}= \begin{cases}1 & \text { if } m_{t}>(1+b) \cdot \operatorname{lbmax}_{t}(l, m) \\ -1 & \text { if } m_{t}<(1-b) \cdot \operatorname{lbmin}_{t}(l, m) \\ S R_{t-1} & \text { otherwise }\end{cases}
$$

The ssh and ssl operators are introduced to simplify the notation of the FI rules. At an interval change at time $t$, for a position initiated at $t_{1}$ (and still held at $t$, with $t_{1}<t$ ), the subsequent high $(s s h)$ and low are $\max \left(m_{t_{1}} \ldots m_{t-1}\right)$ and $\min \left(m_{t_{1}} \ldots m_{t-1}\right)$, respectively. At time $t$ Equation (8) provides a buy or (sell) signal when the return over the last interval breaches the theshold $(b \cdot x)$ from below (above). When at time $t$ a long (short) position is active a neutral signal is provided when the stoploss return 
$\frac{s s h-m_{t}}{s s h}\left(\frac{m_{t}-s s l}{s s l}\right)$ exceeds the threshold $(x \cdot y)$.

$$
F I_{t}= \begin{cases}1 & \text { if } \operatorname{ret}(t, m)>x \\ -1 & \text { if } \operatorname{ret}(t, m)<-x \\ 0 & \text { if } F I_{t-1}=1 \text { and } \frac{m_{t}-s s h}{s s h}<-(x \cdot y) \text { or } \\ & \text { if } F I_{t-1}=-1 \text { and } \frac{m_{t}-s s l}{s s l}>(x \cdot y) \\ F I_{t-1} & \text { otherwise }\end{cases}
$$

Equation (9) provides the CB rule. Here, $I_{C H}(t)$ is the channel indicator at time $t$ which has the value 1 if a channel exists and 0 otherwise. At time $t$ a channel exists if $\operatorname{lbmin}_{t}(l, m)>y \cdot \operatorname{lbmax}_{t}(l, m)$ where the strategy specific parameter $y<1$. Buy (sell) signals are produced when the current midpoint exceeds (falls below) the upper (lower) bound of the channel.

$$
C B_{t}= \begin{cases}1 & \text { if } I_{C H}(t)=1 \text { and } m_{t}>(1+b) \cdot \operatorname{lbmax}(l, m) \\ -1 & \text { if } I_{C H}(t)=1 \text { and } m_{t}<(1-b) \cdot \operatorname{lbmin}(l, m) \\ C B_{t} & \text { otherwise }\end{cases}
$$

Raw signals can take the values $1,-1$, and 0 , corresponding to a long position, short position, and neutral position, respectively. As in Sullivan et al. (1999) two universal parameters are applied to the raw signals of all trading rules in addition to the bandwith parameter $b$. These are a delay parameter $d$ and minimum holding period parameter $h$. The delay parameter ensures that a raw signal $S$ is present for at least $d$ intervals before a trade is initialized, which is then held for at least the minimum holding period of $h$ 
intervals. The rule specific and universal settings used for each trading rule are provided in Appendix A.

\section{Results}

Figure 6 demonstrates the importance of speed for SPY and a capacity level of 100 ETFs. The importance of speed is calculated in percentages relative to the performance of the instantaneous strategy on a daily basis. ${ }^{22}$ Note that strategies with a performance of zero percent are excluded because this makes a relative comparison impossible. Furthermore, if no trading signals are provided implementing a delay has no effect. A Wilcoxon signedrank test is performed to assess whether the performance is significantly different from the instantaneous strategy. The importance of speed for SPY and a capacity level of 100 ETFs stays flat up to $200 \mathrm{~ms}$. After that, the relative performance decreases to about $-3 \%$. Remarkable is that being very fast (10 ms) leads to a worse relative performance than being slightly slower (20 ms up to $200 \mathrm{~ms}$ ).

A distorting factor can be the fact that in Figure 6 strategies with both a positive and a negative performance are included. It is not likely that market participants compete for bad signals and use losing strategies over longer periods of time. Therefore, we expect that speed is more important for profitable strategies. In order to investigate this we split the universe of trading strategies per day up into strategies with a positive and negative per-

\footnotetext{
${ }^{22}$ On day $i$ the average performance per strategy in case of instantaneous execution is compared with the average performance per strategy in case of a delay $d$. The overall importance of speed is then obtained by averaging the daily relative differences over all 186 days in the sample.
} 
formance, when implemented instantaneously. In addition the top (bottom) quantile of strategies with a positive (negative) performance are considered, resulting in a total of four groups of strategies.

When investigating the importance of speed separately for strategies with a positive and negative performance we face a potential selection bias. By selecting all positive strategies, the focus is on stragies for which the individual trades, and the subintervals of individual trades, on average, have a positive return. If a delay $(d)$ is introduced for a trade (with a positive return, entered at time $t_{1}$ and unwinded at time $t_{2}$ ), the subinterval from $t_{1}$ to $t_{1}+d$ is replaced with the interval $t_{2}$ to $t_{2}+d$. Whereas the former interval has, on average, a positive return, this does not necessarily hold for the latter subinterval. Therefore, due to the nature of the selection procedure, one can already expect that introducing a delay for strategies with a positive (negative) performance leads to a lower (higher) performance.

In order to investigate whether we actually measure the impact of speed it is necessary to quantify the impact and magnitude of the selection bias. This is done by implementing the trading signals generated on a certain day on another random day from the sample. The resulting random strategies, which have exactly the same characteristics as the nonrandom strategies, are then subjected to the analysis of the importance of speed ${ }^{23}$ Since the random signals are no true signals it is expected that any importance of speed here is mainly due to the selection bias ${ }^{24}$ The terms 'importance of speed of the

\footnotetext{
${ }^{23}$ The random strategies are similar to the nonrandom strategies in terms of average number of trades and trade duration.

${ }^{24}$ Note that also for the random strategies the importance of speed is still a mix of pure speed and the selection bias. Due to the random nature of the trading strategies
} 
random strategies' and 'selection bias' are used interchangeably, whereas 'the importance of speed' always refers to the importance of speed of nonrandom strategies. In order to test whether the changes in performance are really due to the importance of speed and not a results of the selection bias we construct a $90 \%$ empirical confidence interval by means of a bootstrap with 10,000 resamples. 25

Table 2 provides the total number of strategies considered per rule type as well as the average number of strategies per day with a nonzero, positive, and negative performance. Of the 27,424 strategies considered per day, on average $71.8 \%$ has a nonzero performance. A zero performance is the result of stringent rule settings for which the conditions are not met during a trading day. Of all strategies with a nonzero performance $41.9 \%$ has a positive return. Most successful are the OBV indicators for which $46.9 \%$ of all indicators with a nonzero performance lead to a positive return. This is only $35.4 \%$ for the MSP rules.

The results of the importance of speed for the four groups of strategies (strategies with a positive and negative performance and the top (bottom) quantiles of the strategies with a positive (negative) performance) is provided in Figure 7. As expected, for strategies with a positive performance being

it is unlikely that trade signals actually match true buy or sell moments provided by nonrandom strategies. The main component of the importance of speed in this case is, therefore, the selection bias. Because in some instances the random signals do match true trade signals, in which case it is expected that we measure above all pure speed, the total magnitude of the selection bias is overestimated.

${ }^{25} \mathrm{~A} 90 \%$ confidence level is provided because this corresponds with a $5 \%$ significance level for a one sided test whether the decrease in relative performance in nonrandom strategies due to a delay is larger than the decrease in performance due to the selection bias. 
slow always decreases performance. For both all strategies with a positive return and the top quantile of strategies with a positive return a delay of 200 $\mathrm{ms}, 500 \mathrm{~ms}$, and 1 second lead to a decrease in performance that is significant after accounting for the selection bias. Whereas speed is important when your strategy performs good, subplots (c) and (d) in Figure 7 show that speed has no influence (does not outperform the selection bias) on strategies with a negative performance.

An important determinant of technical trading rule performance is volatility $(\mathrm{Kho}(1996))$. In order to investigate the impact of volatility on the importance of speed we sort the days based on the maximum intraday VIX value and analyse the top and bottom tertile separately ${ }^{26}$ The results for high and low volatility days are presented in Figure 8 and Figure 9, respectively.

For the high volatility days delays of $50 \mathrm{~ms}, 100 \mathrm{~ms}$, and $200 \mathrm{~ms}$ actually have a positive and significant impact on performance. The economic impact is, however, with approximately $0.1 \%$ negligible ${ }^{27}$ Strategies with a negative performance on high volatility days perform significantly worse than instantaneous execution in case of a delay of $10 \mathrm{~ms}$. However, when the delay increases performance improves. The improvement is significant for $500 \mathrm{~ms}$ (all strategies with a negative performance) and $500 \mathrm{~ms}$ and 1 second (the bottom quantile of strategies with a negative return).28

\footnotetext{
${ }^{26}$ Historical VIX data is obtained from http://www.cboe.com/micro/vix/ historical.aspx

${ }^{2 \overline{7}}$ This means that a strategy with an annual return of $5 \%$ will yield 0.5 basispoints more when the delay is $50 \mathrm{~ms}$ to $200 \mathrm{~ms}$ on high volatility days.

${ }^{28}$ The significant improvement in performance for the bottom quantile of strategies with a negative performance suggests that the buy and sell signals of these strategies are provided at truely unfavourable moments for which enough traders actually identified the right (reverse) trade direction. The competition among traders with the right (profitable)
} 
On low volatility days Figure 9 shows that for strategies with a positive return being slow leads to a significant performance reduction for all delay levels except $20 \mathrm{~ms}$. The decline in performance relative to instantanous execution is small at the $10 \mathrm{~ms}$ delay level (about $-0.1 \%$ ) but increases to about $-1.0 \%(-2.0 \%)$ for a delay of $500 \mathrm{~ms}$ (1 second). Although statistically significant, the economic impact of speed is limited. For example, for a strategy that generates an annual return of $5.0 \%$ you lose 10 basispoints by being 1 second slower.

With all investments of the financial sector in information technology it is expected that speed becomes more important over time. We investigate this formally by regressing the relative importance of speed on a time trend for all strategies with a positive performance. Figure 10 provides the coefficient of the regression for all delay levels in the lower left corner of the plots which also provide the relative importance of speed over time for (solid lines) and the average importance of speed during the month (dashed lines) ${ }^{29}$ A one-sided t-test is performed to check whether the coefficient is significantly smaller than zero. For a $10 \mathrm{~ms}$ delay speed becomes more important over time at the $10 \%$ significance level, whereas for all other delays this is the case at the $1 \%$ level. The coefficient for, for example, a delay level of $50 \mathrm{~ms}$ is $-0.0031 \%$, which indicates that each day an additional $0.0031 \%$ is lost relatively to an instantaneous strategy. This is $0.78 \%$ per year of 252 trading days. For a

signal results in the fact that traders with the wrong (nonprofitable) signal trade against better prices when they are slow.

${ }^{29}$ Note that the figure for a delay level of $20 \mathrm{~ms}$ is not included. The (insignificant) coefficient in this case is -0.0006 . 
delay of $500 \mathrm{~ms}$, and 1 second this is $1.97 \%$ and $3.30 \%$, respectively ${ }^{30}$

\section{Robustness}

\subsection{Other Capacity Levels}

Figure 11 presents the importance of speed for SPY on low volatility days for capacity levels of 100, 500, 1,000, and 5,000 ETFs. Although speed is always most important for a capacity level of 100 ETFs, the difference between the capacity levels is small indicating a stable and highly liquid trading environment. For strategies with a positive performance a capacity level of 500 ETFs leads to the same conclusion as for 100 ETFs. In case of a capacity level of 1,000 ETFs, a delay of $20 \mathrm{~ms}$ now leads to a significant reduction in performance, whereas this is no longer the case for the $50 \mathrm{~ms}$ delay level. For a capacity level of 5,000 ETFs delay levels of $100 \mathrm{~ms}$ and up lead to a significantly lower return compared to instantaneous execution. The (unreported) results in case of all days and days with high volatility also confirm that the importance of speed is robust with respect to the capacity level of the strategies.

\subsection{Other Measures for the Importance of Speed}

In addition to the importance of speed as the relative difference in performance per strategy per day for different delay levels we compute several

\footnotetext{
${ }^{30}$ The regression on a time trend is not performed for days with high or low volatility due to clustering of high and low volatility days. The analysis for the top quantile of strategies with a positive performance results in the coefficients $-0.0005,-0.0025,-0.0019,-0.0029$, $-0.0055,-0.0075$ for the $10 \mathrm{~ms}, 50 \mathrm{~ms}, 100 \mathrm{~ms}, 200 \mathrm{~ms}, 500 \mathrm{~ms}$, and 1 second delay level, respectively. The coefficients for the $10 \mathrm{~ms}$ and $200 \mathrm{~ms}$ delay level are significant at the $10 \%$ level, whereas all other coefficients are significant at either the $1 \%$ or $5 \%$ significance level.
} 
alternatives. The first alternative is the relative importance of speed per rule per day. The difference between this measure and the original method is that for the relative importance of speed per rule per day we first compute the relative importance of speed per rule (the performance of a trading rule for a delay level with respect to the performance of the same trading rule with instantaneous execution). The relative importance of speed per rule is then averaged over the day and, subsequently, sample period. Because the relative importance of speed per rule per day has potentially many extreme values (because of rules with an instantaneous performance close to zero) we also consider a truncated relative performance of speed per rule per day (where relative differences larger (smaller) than 100\% (-100\%) are truncated to $100 \%$ and $-100 \%$ ). Besides the relative importance of speed we also look at the absolute importance of speed (in annualized basispoints (bps)). This measure is the same for a computation per rule per day and per day. Finally, we also provide a nonparametric measure of the importance of speed by simply comparing the number of increases and decreases in performance resulting from introducing a delay.

The results for the four alternative measures of the importance of speed on days with low volatility are provided in the internet appendix. It appears that for every measure the conclusions for strategies with a positive performance is the same for delay levels of $100 \mathrm{~ms}$ and up. This also holds for a delay level of $10 \mathrm{~ms}$ for all measures, except the nonparametric version. The main difference between the different measures is the magnitude of the importance of speed. For example, the reduction in performance in terms of relative importance of speed per day at a delay of 1 second is about $2 \%$. When 
using the relative importance of speed per rule per day (or its truncated counterpart) this is more than $15 \%(2.75 \%)$. The absolute importance of speed in this case is -150 bps $(-1.50 \%)$ per year.

\subsection{Other ETFs and Intervals}

To investigate whether the results for the 60 second interval of SPY also hold in general we determine the importance of speed also for the two other ETFs (QQQQ and IWM) and trading intervals of 30 seconds and 5 minutes ${ }^{31}$ Table 3 presents the results of this analysis for all strategies with a positive performance on low volatility days. Significant values (outside the $90 \%$ confidence interval around the selection bias) are marked with a *.

For the 30 seconds (5 minute) interval of SPY a delay of $100 \mathrm{~ms}$ (200 ms) or more leads to a significantly lower relative performance. This also holds for all intervals of SPY at a delay level of $10 \mathrm{~ms}$. The impact of speed for SPY is highest for the 30 second interval (-2.3047\%), followed by the 60 second $(-2.0263 \%)$ and 5 minute $(-1.4735 \%)$ intervals. In the case of QQQQ a significantly lower return occurs at the $10 \mathrm{~ms}$ and $20 \mathrm{~ms}$ delay levels of the 60 second interval, the $20 \mathrm{~ms}$ delay level of the 5 minute interval, and for delay levels of $500 \mathrm{~ms}$ and up for all intervals. A delay of $50 \mathrm{~ms}$ (100 ms) or more is enough for a significant decrease in strategy performance for the 30 seconds (60 seconds) interval of IWM. At the 5 minute interval of IWM a 1 second delay is necessary to lead to a significant reduction in the strategy return. For delay levels of $500 \mathrm{~ms}$ and 1 second speed is more important for

\footnotetext{
${ }^{31}$ Some of the rule specific or universal parameters for the 30 second and 5 minute intervals are different from the settings used for the interval of 60 seconds and can also be found in Appendix A
} 
QQQQ and IWM compared to SPY. For example, at the $500 \mathrm{~ms}$ (1 second) delay level of the 60 second interval the reduction in performance is $-2.0189 \%$ $(-3.2825 \%)$ for QQQQ, $-1.2761 \%(-2.5928 \%)$ for IWM compared to $-0.9978 \%$ $(-2.0263 \%)$ for SPY.

The results over all days in the sample and on high volatility days can be found in the internet appendix. Compared to the results over all days (see Figure 7) the most noteworthy differences for other intervals and ETFs are that for IWM the reduction in performance due to delays is significant for lags of $500 \mathrm{~ms}$ and up. For QQQQ a $10 \mathrm{~ms}$ and $500 \mathrm{~ms}$ delay for the 60 second interval as well as a $20 \mathrm{~ms}$ and 1 second delay for all intervals lead to a significant reduction in performance. The 30 second ( 5 minute) interval of SPY exhibits a significant reduction in returns when the delay is $200 \mathrm{~ms}$ (500 $\mathrm{ms}$ and 1 second).

The main differences for other intervals and ETFs on high volatility days is that the significant and positive impact of delays of $50 \mathrm{~ms}, 100 \mathrm{~ms}$, and 200 ms on return (see Figure 8) for the 60 second interval of SPY is not found for QQQQ and IWM (exept for the $100 \mathrm{~ms}$ delay level of the 60 second interval of IWM). In fact, for IWM and QQQQ all other significant values for the importance of speed suggest that also on high volatility days a delay in execution leads to a lower return.

\subsection{Other ETFs and Importance of Speed over Time}

Table 4 provides the coefficients of a regression of the importance of speed on a time trend (over all 186 trading days in the sample) for all positive strategies of the 30 second, 60 second, and 5 minute intervals for SPY, QQQQ, and IWM. Whereas for SPY the coefficients are significant (at either a $5 \%$ or 
$1 \%$ significance level) for all delay levels above $50 \mathrm{~ms}$, this is not the case for QQQQ and IWM. For QQQQ speed becomes more important over time only for delay levels of $500 \mathrm{~ms}$ and 1 second. In the case of IWM the importance of speed increases over time for the 30 (60) second intervals for delay levels of $50 \mathrm{~ms}(100 \mathrm{~ms})$ and up, whereas for the 5 minute interval this is only the case for the 1 second delay level. When either IWM or QQQQ exhibits a significant increase of the importance of speed over time this is most of the times a slightly larger increase compared to the same trading interval and delay combination of SPY. Of the three different intervals the importance of speed increases least for the 5 minute interval. For example, for IWM at the 1 second delay level the coefficient of the 30 second interval is $-0.0205 \%$. This is only $-0.0106 \%$ for the 5 minute interval. The coefficients of the importance of speed for the $20 \mathrm{~ms}$ and $100 \mathrm{~ms}$ (10 ms and $50 \mathrm{~ms}$ ) of the 30 second interval of QQQQ (5 minute interval of IWM) suggest a significant decrease of the importance of speed over time at a 10\% significance level.

\section{Conclusion}

This paper investigates the importance of speed on the performance of basic technical trading rules for three highly liquid ETFs (S\&P 500 (SPY), NASDAQ 100 (QQQQ), and Russell 2000 (IWM)) traded on NASDAQ over

the period January 6, 2009 up to September 30, 2009. This is done by comparing the performance of 27,424 trading rules from 7 rule families when trading signals get executed immediately at the interval change with trading signals that get executed after lags of $10 \mathrm{~ms}, 20 \mathrm{~ms}, 50 \mathrm{~ms}, 100 \mathrm{~ms}, 200 \mathrm{~ms}$, $500 \mathrm{~ms}$ and 1 second. The importance of speed is calculated relative to the 
performance of immediate execution at the interval change.

We find that speed is important for trading rules with a positive performance. Trading strategies where signals are executed $200 \mathrm{~ms}$ after the interval change significantly underperform strategies with instantaneous execution. The effect is stronger on days with low volatility (in this case a delay of $50 \mathrm{~ms}$ already leads to a significantly lower return with respect to instantaneous strategies) and when one trades small amounts of ETFs. Although the impact of speed is statistically significant, the economic impact is limited. A regression of the importance of speed on a time trend indicates that speed becomes more important over time, suggesting that speed could eventually be a major factor in the performance of simple technical trading rules. All results are robust with respect to the choice of the interval, ETF, and the measure used for the importance of speed.

The fact that speed is only important for strategies with a positive performance can be explained by fierce competition for good signals, whereas there is no competition (no need for speed) for strategies with negative returns and poor signals. It must be noted that the strategies under consideration are standard interval based trading rules. It is likely that speed is already much more important for more complicated interval based trading rules or event based trading rules triggered by certain order book events.

In addition to the importance of speed we investigate market activity, which is measured by means of message intensity. Of the three ETFs, most activity takes place in SPY. The distribution of messages over the day is not constant and follows a U-shape with a large peak at 10:00AM and small peaks during whole and half hours. Within the hour (minute) clear periodic 
patterns are identified with the largest spikes at the change of the hour (minute). The distribution of messages within the second shows large peaks at 5 and 50 milliseconds. It is likely that the peaks are caused by traders with the same technological level at the same geographic location. About $50 \%$ of all message activity consists orders added and removed from the orderbook within 1 second. Furthermore, for SPY at least 50\% (15\%) of all nonmarketable limit orders are added and removed from the orderbook within 1 second (10 ms). Most of this activity (at least 30\%) takes place at the best bid or ask quote. About $85 \%$ of all improvements to the best bid or ask quote are either removed or fully executed within 1 second for SPY (this is about 60\% for QQQQ and 70\% for IWM).

\section{Acknowledgements}

The authors would like to thank NASDAQ for providing the data for this research, Eran Raviv and Karolina Scholtus for helpful comments and suggestions, and Justinas Brazys for providing data on macroeconomic announcements.

\section{References}

Brock, W., Lakonishok, J., LeBaron, B., 1992. Simple technical trading rules and the stochastic properties of stock returns. The Journal of Finance 47, 1731.

Brogaard, J.A., 2010. High frequency trading and its impact on market quality. Working paper, Northwestern University. 
Chaboud, A., Chiquoine, B., Hjalmarsson, E., Vega, C., 2009. Rise of the machines: Algorithmic trading in the foreign exchange market. FRB International Finance Discussion Paper No. 980.

Cialenco, I., Protopapadakis, A., 2011. Do technical trading profits remain in the foreign exchange market? Evidence from 14 currencies. Journal of International Financial Markets, Institutions and Money 21, 176-206.

Ende, B., Uhle, T., Weber, M.C., 2011. The impact of a millisecond: Measuring latency effects in securities trading, in: Wirtschaftinformatik Proceedings 2011, pp. 27-37.

Fama, E., Blume, M., 1966. Filter rules and stock-market trading. Journal of Business 39, 226-241.

Garvey, R., Wu, F., 2009. Intraday time and order execution quality dimensions. Journal of Financial Markets 12, 203-228.

Gençay, R., 1998. The predictability of security returns with simple technical trading rules. Journal of Empirical Finance 5, 347-359.

Gomber, P., Arndt, B., Lutat, M., Uhle, T., 2011. High-frequency trading. Working paper, University of Frankfurt.

Hasbrouck, J., Saar, G., 2009. Technology and liquidity provision: The blurring of traditional definitions. Journal of Financial Markets 12, 143172.

Hasbrouck, J., Saar, G., 2011. Low-latency trading. Working paper, Johnson School Research Paper Series No. 35-2010. 
Hendershott, T., Jones, C.M., Menkveld, A.J., 2011. Does algorithmic trading improve liquidity? Journal of Finance 66, 1-33.

Hendershott, T., Riordan, R., 2011. Algorithmic trading and information. Working paper, University of California at Berkeley.

Hsu, P.H., Kuan, C.M., 2005. Reexamining the profitability of technical analysis with data snooping checks. Journal of Financial Econometrics 3, 606-628.

Kho, B.C., 1996. Time-varying risk premia, volatility, and technical trading rule profits: Evidence from foreign currency futures markets. Journal of Financial Economics 41, 249-290.

Kirilenko, A., Kyle, A.S., Samadi, M., Tuzun, T., 2011. The flash crash: The impact of high frequency trading on an electronic market. Working paper, University of Maryland.

Lehmann, B.N., Modest, D.M., 1994. Trading and liquidity on the Tokyo stock exchange: A bird's eye view. The Journal of Finance 49, 951.

Marshall, B., Cahan, R., Cahan, J., 2008. Does intraday technical analysis in the U.S. equity market have value? Journal of Empirical Finance 15, 199-210.

McInish, T.H., Wood, R.A., 1992. An analysis of intraday patterns in bid/ask spreads for NYSE stocks. The Journal of Finance 47, 753.

Menkhoff, L., 2010. The use of technical analysis by fund managers: International evidence. Journal of Banking and Finance 34, 2573-2586. 
Menkhoff, L., Taylor, M.P., 2007. The obstinate passion of foreign exchange professionals: Technical analysis. Journal of Economic Literature 45, 936972.

Moallemi, C.C., Saglam, M., 2010. The cost of latency. Working paper, Columbia University.

Neely, C., Weller, P., Dittmar, R., 1997. Is technical analysis in the foreign exchange market profitable? A genetic programming approach. Journal of Financial and Quantitative Analysis 32, 405-426.

O'Hara, M., 2010. What is a quote? The Journal of Trading 5, 10-16.

Olson, D., 2004. Have trading rule profits in the currency markets declined over time? Journal of Banking and Finance 28, 85-105.

Park, C.H., Irwin, S.H., 2007. What do we know about the profitability of technical analysis? Journal of Economic Surveys 21, 786-826.

Prix, J., Loistl, O., Huetl, M., 2007. Algorithmic trading patterns in Xetra orders. The European Journal of Finance 13, 717-739.

Qi, M., Wu, Y., 2006. Technical trading-rule profitability, data snooping, and reality check: Evidence from the foreign exchange market. Journal of Money, Credit, and Banking 38, 2135-2158.

Schulmeister, S., 2009. Profitability of technical stock trading: Has it moved from daily to intraday data? Review of Financial Economics 18, 190-201. 
Sullivan, R., Timmermann, A., White, H., 1999. Data-snooping, technical trading rule performance, and the bootstrap. The Journal of Finance 54, $1647-1691$.

Szakmary, A.C., Shen, Q., Sharma, S.C., 2010. Trend-following trading strategies in commodity futures: A re-examination. Journal of Banking and Finance 34, 409-426. 


\section{Figures and Tables}

Table 1: Average message intensity $(\times 1000)$ per day per message type.

\begin{tabular}{crrrrrrrrr}
\hline & $\mathrm{A}$ & $\mathrm{C}$ & \multicolumn{1}{c}{$\mathrm{D}$} & $\mathrm{E}$ & $\mathrm{F}$ & $\mathrm{P}$ & $\mathrm{U}$ & $\mathrm{X}$ & TOTAL \\
\hline SPY & & & & & & & & & \\
Mean & 2555.41 & 18.38 & 2334.34 & 286.93 & 4.97 & 7.47 & 622.15 & 83.10 & 5698.69 \\
Median & 1698.24 & 10.51 & 1519.78 & 232.71 & 3.48 & 6.33 & 547.22 & 55.57 & 4449.97 \\
\% of Total & 43.03 & 0.27 & 38.97 & 5.37 & 0.09 & 0.14 & 16.69 & 1.19 & 100.00 \\
Stdev & 1757.31 & 16.71 & 1641.57 & 143.84 & 3.76 & 4.90 & 359.33 & 123.87 & 3420.39 \\
& & & & & & & & & \\
QQQQ & & & & & & & & & \\
Mean & 1470.43 & 4.06 & 1418.57 & 79.13 & 8.36 & 3.02 & 584.44 & 47.27 & 3414.19 \\
Median & 1111.62 & 3.92 & 1056.89 & 75.32 & 7.03 & 2.51 & 495.14 & 35.10 & 2920.20 \\
\% of Total & 41.86 & 0.11 & 40.21 & 2.52 & 0.26 & 0.10 & 20.61 & 1.43 & 100.00 \\
Stdev & 861.30 & 3.44 & 845.00 & 30.24 & 3.97 & 2.20 & 806.71 & 30.36 & 1779.30 \\
& & & & & & & & & \\
IWM & & & & & & & & & \\
Mean & 1770.63 & 4.30 & 1711.37 & 76.47 & 2.69 & 1.99 & 373.20 & 101.24 & 3913.49 \\
Median & 1506.77 & 3.44 & 1452.05 & 71.80 & 2.26 & 1.77 & 338.87 & 95.67 & 3628.53 \\
\% of Total & 44.23 & 0.10 & 42.72 & 1.97 & 0.07 & 0.05 & 12.22 & 2.85 & 100.00 \\
Stdev & 787.18 & 3.32 & 764.86 & 31.70 & 1.50 & 1.01 & 126.44 & 36.08 & 1473.12 \\
\hline
\end{tabular}

This Table shows per ETF (SPY, QQQQ, and IWM), the mean, median, and standard deviation of the number of messages per message type per day in thousands. The percentage of total message activity per day attributed to a certain message type is provided by $\%$ of Total. The message types, denoted by capital letters are: A; add order message (no market participant identifier), F; add order message (with market participant identifier), C; order executed message (with price), E; order executed message (without price), D; order delete message, X; order cancel message, U; order replace message, $\mathrm{P}$; trade of nondisplayable order message. 
Table 2: Number of strategies included for a capacity level of 100 ETFs.

\begin{tabular}{crrrrrr}
\hline & TOT & \multicolumn{1}{c}{ ALL } & \multicolumn{1}{c}{ POS } & POS25 & \multicolumn{1}{c}{ NEG } & NEG25 \\
\hline MA & 4608.0 & 3686.7 & 1475.1 & 368.4 & 2211.6 & 552.5 \\
SR & 2288.0 & 1919.0 & 852.3 & 212.7 & 1066.8 & 266.4 \\
FI & 1600.0 & 967.9 & 346.7 & 86.3 & 621.2 & 154.9 \\
CBO & 2800.0 & 2230.7 & 1002.6 & 250.5 & 1228.1 & 306.9 \\
OBV & 5376.0 & 4734.0 & 2220.7 & 554.8 & 2513.3 & 627.9 \\
MSP & 5376.0 & 2009.0 & 711.9 & 177.6 & 1297.1 & 323.9 \\
MSV & 5376.0 & 4144.3 & 1635.2 & 408.4 & 2509.0 & 626.9 \\
SUM & 27424.0 & 19691.6 & 8244.4 & 2058.7 & 11447.2 & 2859.5 \\
\hline
\end{tabular}

This table shows the number of strategies per rule type included in the analysis for a capacity of 100 ETFs. TOT provides the total number of strategies considered per rule type per day. ALL provides the average number of strategies per rule type per day with a nonzero performance, whereas POS (NEG) and POS25 (NEG25) provide the average number of rules with positive (negative) performance and the number of rules in the top (bottom) quantile of the positive (negative) performing rules per day, respectively. 
Table 3: The importance of speed (in \%) for different delay levels, intervals, and ETFs on days with low volatility for a capacity level of 100 ETFs.

\begin{tabular}{|c|c|c|c|c|c|c|c|c|}
\hline & & $10 \mathrm{~ms}$ & $20 \mathrm{~ms}$ & $50 \mathrm{~ms}$ & $100 \mathrm{~ms}$ & $200 \mathrm{~ms}$ & $500 \mathrm{~ms}$ & $1 \mathrm{sec}$ \\
\hline \multirow[t]{3}{*}{ SPY } & $30 \mathrm{sec}$ & $-0.0863^{*}$ & -0.0075 & -0.1400 & $-0.1630^{*}$ & $-0.4477^{*}$ & $-1.0889^{*}$ & $-2.3047^{*}$ \\
\hline & $60 \mathrm{sec}$ & $-0.1049^{*}$ & -0.0640 & -0.2650 & $-0.2985^{*}$ & $-0.4916^{*}$ & $-0.9978^{*}$ & $-2.0263^{*}$ \\
\hline & $5 \min$ & $-0.0337^{*}$ & -0.0406 & -0.1959 & -0.1599 & $-0.4507^{*}$ & $-0.8162^{*}$ & $-1.4735^{*}$ \\
\hline \multirow[t]{3}{*}{ QQQQ } & $30 \mathrm{sec}$ & -0.0147 & -0.0104 & 0.1376 & 0.0922 & -0.2149 & $-1.4548^{*}$ & $-2.7177^{*}$ \\
\hline & $60 \mathrm{sec}$ & $-0.0530^{*}$ & $-0.0489^{*}$ & 0.0625 & -0.0488 & -0.3450 & $-2.0189^{*}$ & $-3.2825^{*}$ \\
\hline & $5 \min$ & -0.0751 & $-0.0754^{*}$ & -0.0556 & -0.1412 & -0.5000 & $-1.3618^{*}$ & $-2.1126^{*}$ \\
\hline \multirow[t]{3}{*}{ IWM } & $30 \mathrm{sec}$ & 0.0343 & -0.0171 & $-0.1081^{*}$ & $-0.2705^{*}$ & $-0.4959^{*}$ & $-1.7177^{*}$ & $-3.2670^{*}$ \\
\hline & $60 \mathrm{sec}$ & 0.0577 & 0.0492 & -0.0259 & $-0.2563^{*}$ & $-0.4165^{*}$ & $-1.2761^{*}$ & $-2.5928^{*}$ \\
\hline & $5 \min$ & 0.1026 & 0.0739 & 0.0271 & -0.0355 & -0.2233 & -0.6398 & $-1.9559^{*}$ \\
\hline
\end{tabular}

This table provides the importance of speed (in \% with respect to instantaneous execution) for all trading rules with a positive performance on low volatility days (the bottom tercile for the intraday high of the VIX over the period January 6, 2009 to September 30, 2009) and a capacity level of 100 ETFs. The importance of speed is provided for seven different delay levels (10 $\mathrm{ms}$ up to 1 second), three ETFs (SPY, IWM, and QQQQ) and three trading frequencies (30 seconds, 60 seconds, and 5 minutes). For different levels of delay (between the moment a trading interval changes and the moment a signal is implemented) in milliseconds (ms), the percentage value indicates how the performance of strategies with instantaneous execution (a delay of $0 \mathrm{~ms}$ ) changes when execution is delayed. Values with a $*$ are significantly different from the selection bias (importance of speed for random strategies). 
Table 4: The importance of speed through time for different delay levels, intervals, and ETFs for a capacity level of 100 ETFs.

\begin{tabular}{ccccccccc}
\hline \multicolumn{1}{c}{ SPY } & & $10 \mathrm{~ms}$ & $20 \mathrm{~ms}$ & $50 \mathrm{~ms}$ & $100 \mathrm{~ms}$ & $200 \mathrm{~ms}$ & $500 \mathrm{~ms}$ & $1 \mathrm{sec}$ \\
& $60 \mathrm{sec}$ & -0.0004 & -0.0002 & $-0.0016^{* *}$ & $-0.0024^{* *}$ & $-0.0042^{* * *}$ & $-0.0091^{* * *}$ & $-0.0161^{* * *}$ \\
& $5 \mathrm{~min}$ & -0.0001 & -0.0005 & $-0.0027^{* * *}$ & $-0.0022^{* *}$ & $-0.0042^{* * *}$ & $-0.0060^{* *}$ & $-0.0092^{* * *}$ \\
& & & & & & & & \\
QQQQ & $30 \mathrm{sec}$ & 0.0003 & $0.0009^{*}$ & 0.0019 & $0.0026^{*}$ & 0.0007 & $-0.0090^{* *}$ & $-0.0179^{* * *}$ \\
& $60 \mathrm{sec}$ & -0.0002 & -0.0004 & 0.0002 & 0.0004 & -0.0005 & $-0.0116^{* * *}$ & $-0.0193^{* * *}$ \\
& $5 \mathrm{~min}$ & -0.0000 & -0.0000 & 0.0004 & -0.0005 & -0.0018 & $-0.0075^{* * *}$ & $-0.0126^{* * *}$ \\
& & & & & & & & \\
IWM & $30 \mathrm{sec}$ & 0.0000 & -0.0001 & $-0.0012^{*}$ & $-0.0025^{* *}$ & $-0.0041^{* *}$ & $-0.0107^{* * *}$ & $-0.0205^{* * *}$ \\
& $60 \mathrm{sec}$ & 0.0002 & 0.0002 & -0.0004 & $-0.0023^{* *}$ & $-0.0033^{* *}$ & $-0.0071^{* * *}$ & $-0.0159^{* * *}$ \\
& $5 \mathrm{~min}$ & $0.0008^{*}$ & 0.0006 & $0.0011^{*}$ & 0.0009 & 0.0001 & -0.0003 & $-0.0106^{* *}$ \\
\hline
\end{tabular}

This table provides the regression coefficients of the importance of speed regressed on a time trend for three ETFs (SPY, QQQQ, and IWM), three different trading frequencies (30 seconds, 60 seconds, and 1 minute), and seven delay levels (10 ms up to 1 second) for a capacity level of 100 ETFs for all trading days in the sample. Values marked with a $(*),\left({ }^{*}\right)$, or $\left({ }^{* *}\right)$ mark the coefficients which are significant at the $10 \%, 5 \%$, and $1 \%$ level (based on a one-sided t-test), respectively. 
Figure 1: Average total message intensity for SPY per minute for each day over the period January 6, 2009 to September 30, 2009

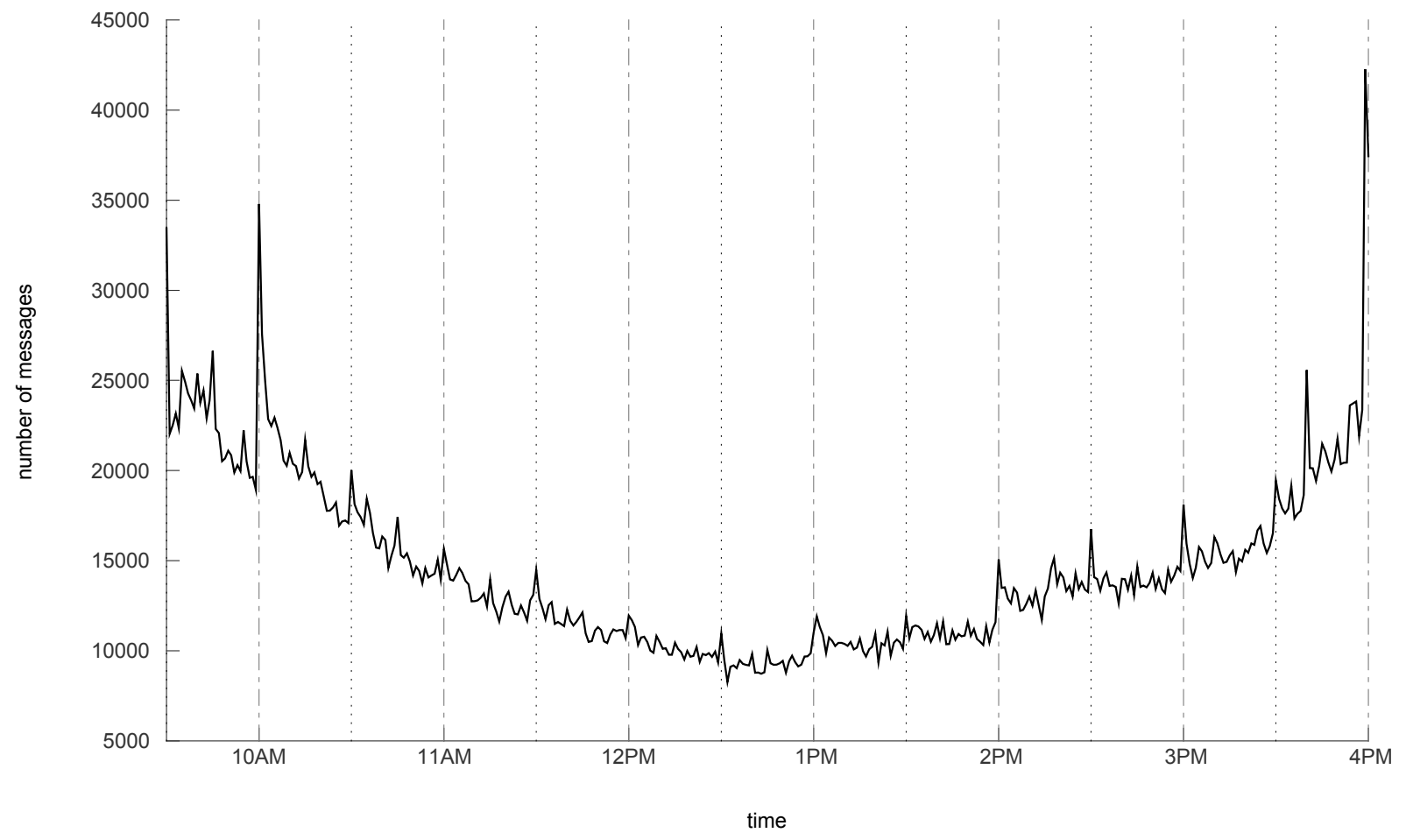


Figure 2: Average total message intensity for SPY per minute for each hour excluding the first 90 and last 60 minutes of the trading day over the period January 6, 2009 to September 30, 2009

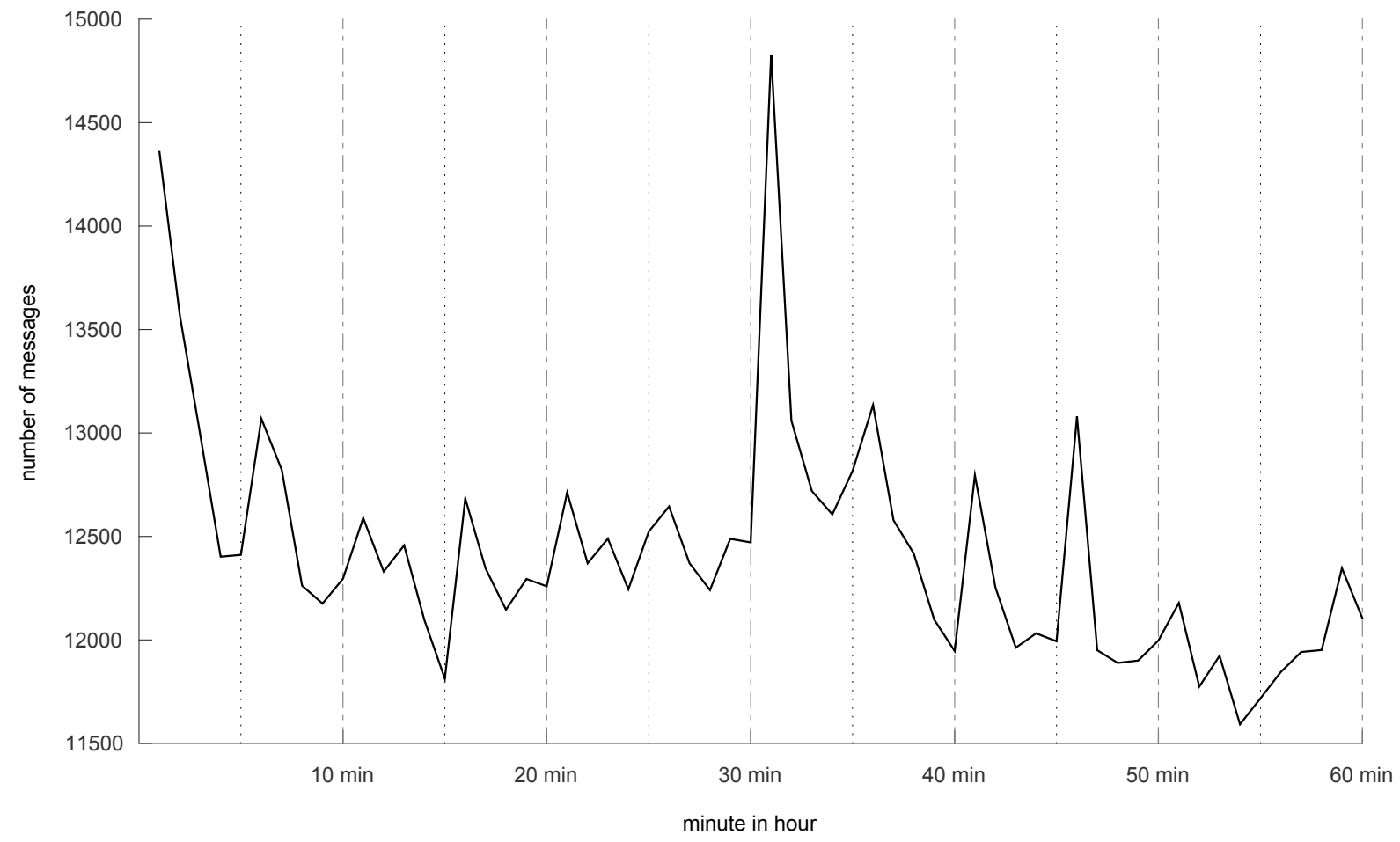


Figure 3: Average total message intensity for SPY per second for each minute excluding the first 90 and last 60 minutes of the trading day over the period January 6, 2009 to September 30, 2009

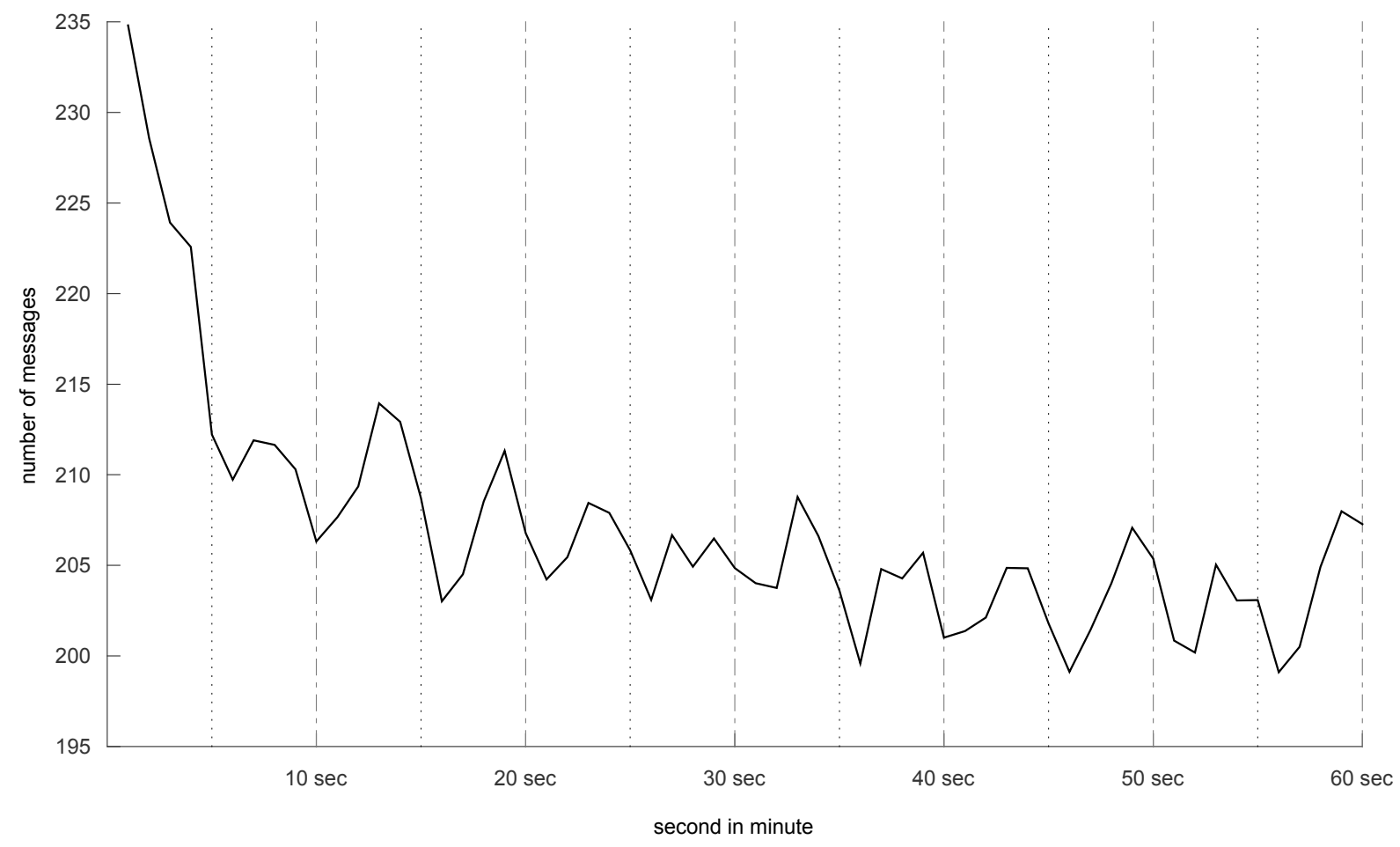


Figure 4: Average total message intensity for SPY per millisecond for each second excluding the first 90 and last 60 minutes of the trading day over the period January 6, 2009 to September 30, 2009

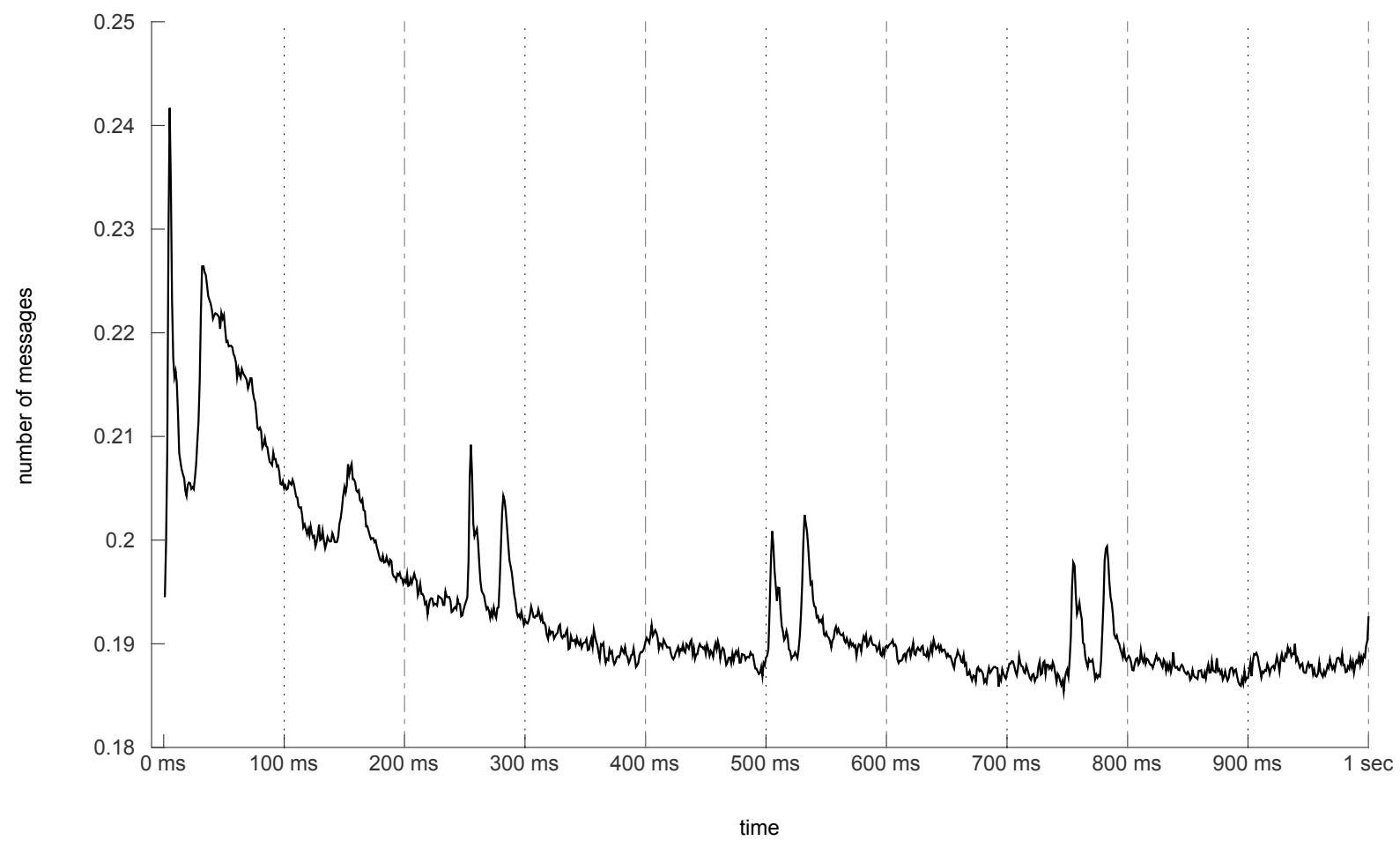


Figure 5: Percentage of total message activity over the period January 6, 2009 to September 30, 2009 during normal trading hours due to fleeting orders (a), (b) the distribution of the point of entry of fleeting orders in the orderbook where $0(-1)$ and $x$ indicate additions at the best bid or ask quote, before the best bid or ask quote, and $x$ cents behind the best bid or ask quote (with $1 \leq x \leq 23$ ), respectively. Subplot (c) provides the percentage of all improvements to the best bid or ask quote that dissapear (either due to cancellations or executions) within $10 \mathrm{~ms}$ up to 1 second, whereas (d) shows the percentage of missed opportunities within $10 \mathrm{~ms}$ up to 1 second. A missed opportunity is an add order message which is an improvement to the best bid or ask that, when it is either removed or executed, leaves a worse price in the orderbook.

(a) \% FLEETS OF LIMIT ORDERS

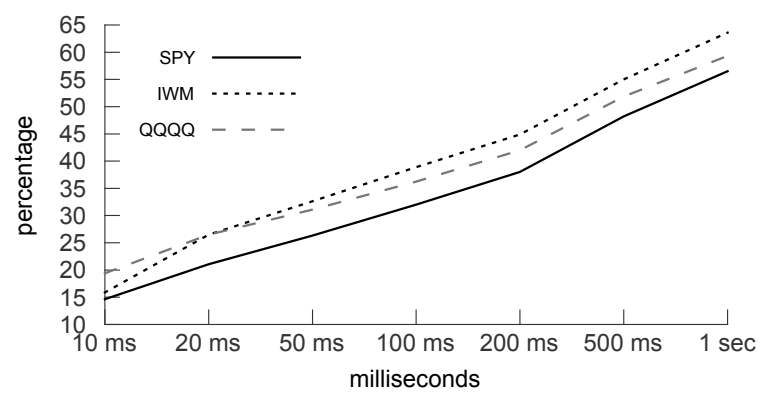

(c) \% OF BBA IMPROVEMENTS THAT DISSAPEAR

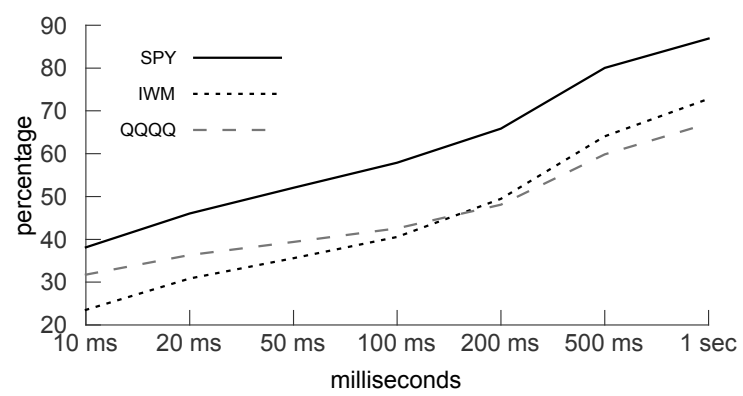

(b) \% FLEETS WRT BBA (50ms)

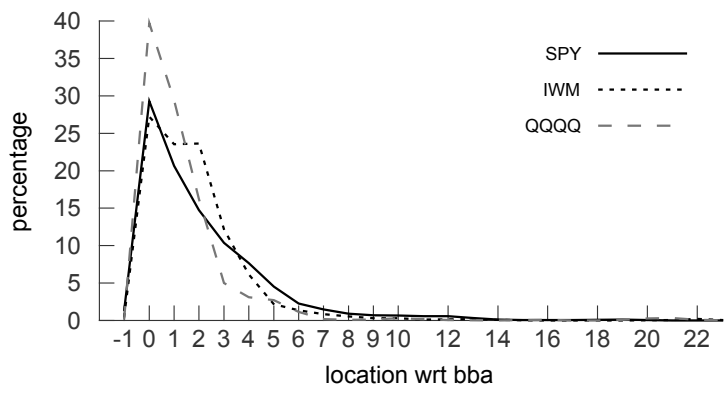

(d) \% OF MISSED OPPORTUNITIES

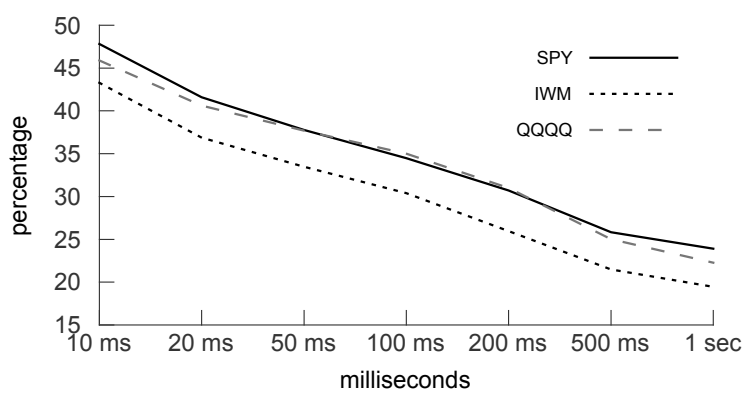


Figure 6: The importance of speed for all trading strategies and a trade capacity of 100 ETFs. For different levels of delay (between the moment a trading interval changes and the moment a trade signal is constructed and executed) in milliseconds (ms), the percentage value indicates how the performance of strategies with instantaneous execution (a delay of $0 \mathrm{~ms}$ ) changes when execution is delayed. For each capacity and delay level a two-sided Wilcoxon signed-rank test is performed to check whether the relative performance is significantly different from instantaneous execution. Significant values at the 10\%, $5 \%$, and $1 \%$ level are indicated by a $\odot, \bullet$, and $*$, respectively.

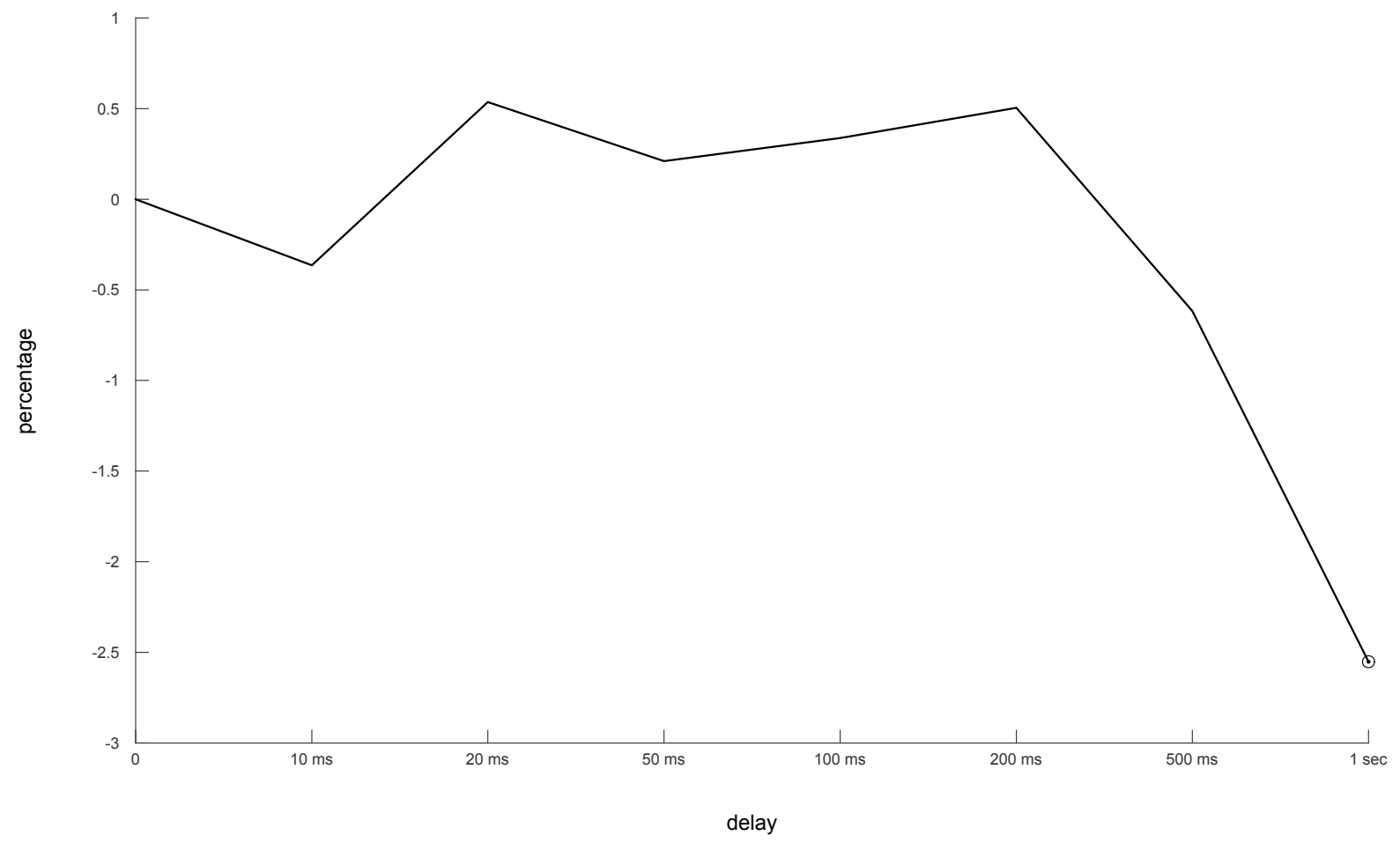


Figure 7: The importance of speed for all trading strategies with a positive (a) and negative (c) performance as well as the importance of speed for the top (bottom) quartile of the strategies with a positive (negative) performance in (b) and (d), respectively. All strategies are implemented with a capacity level of 100 ETFs. For different levels of delay (between the moment a trading interval changes and the moment a trade signal is constructed and executed) in milliseconds (ms), the percentage value (corresponding to the solid dark line) indicates how the performance of strategies with instantaneous execution (a delay of 0 ms) changes when execution is delayed. The selection bias and corresponding $90 \%$ bootstrapped confidence interval is provided by the solid light line and dashed lines, respectively. In case the importance of speed is significant this is indicated by a $*$.

(a) ALL POSITIVE

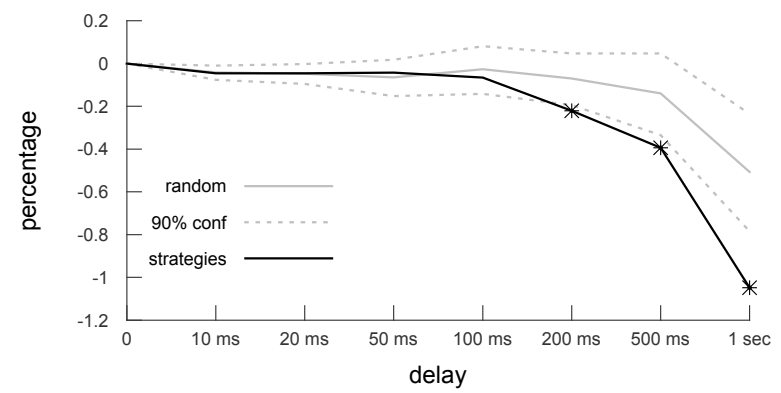

(c) ALL NEGATIVE

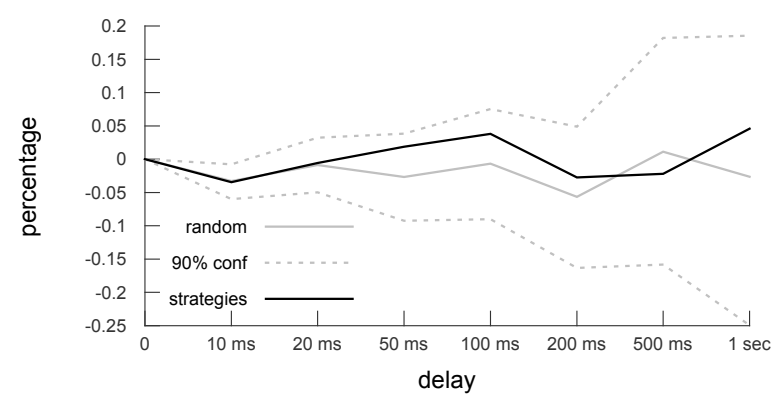

(b) TOP $25 \%$ POSITIVE

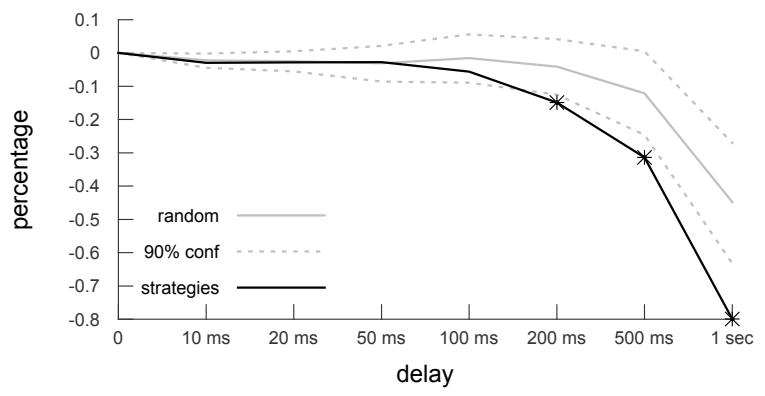

(d) BOTTOM $25 \%$ NEGATIVE

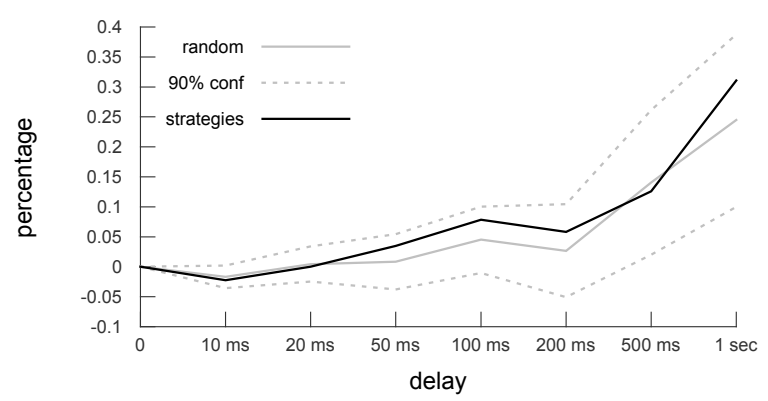


Figure 8: The importance of speed on the days with high volatility (the top tercile for the intraday high of the VIX over the period January 6, 2009 to September 30, 2009) for all trading strategies with a positive (a) and negative (c) performance as well as the importance of speed for the top (bottom) quartile of the strategies with a positive (negative) performance in (b) and (d), respectively. All strategies are implemented with a capacity level of 100 ETFs. For different levels of delay (between the moment a trading interval changes and the moment a trade signal is constructed and executed) in milliseconds (ms), the percentage value (corresponding to the solid dark line) indicates how the performance of strategies with instantaneous execution (a delay of $0 \mathrm{~ms}$ ) changes when execution is delayed. The selection bias and corresponding $90 \%$ bootstrapped confidence interval is provided by the solid light line and dashed lines, respectively. In case the importance of speed is significant this is indicated by a $*$.

(a) ALL POSITIVE

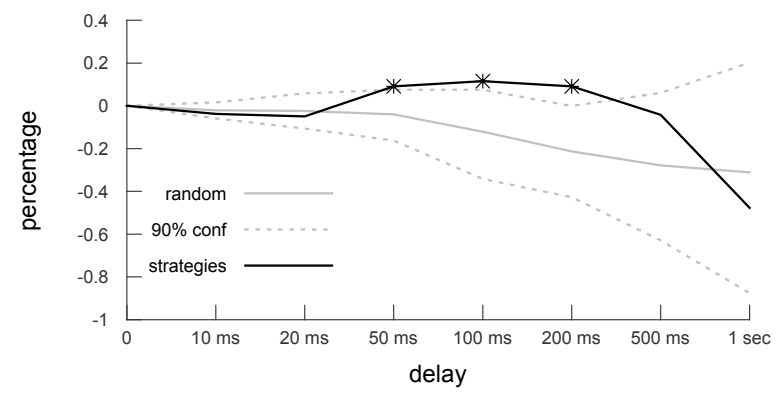

(c) ALL NEGATIVE

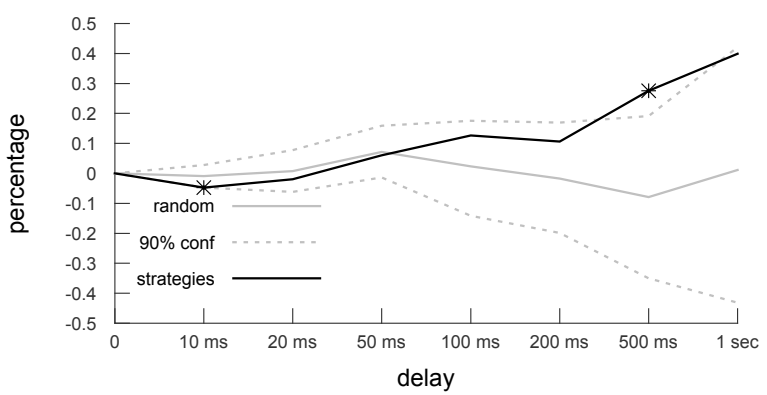

(b) TOP 25\% POSITIVE

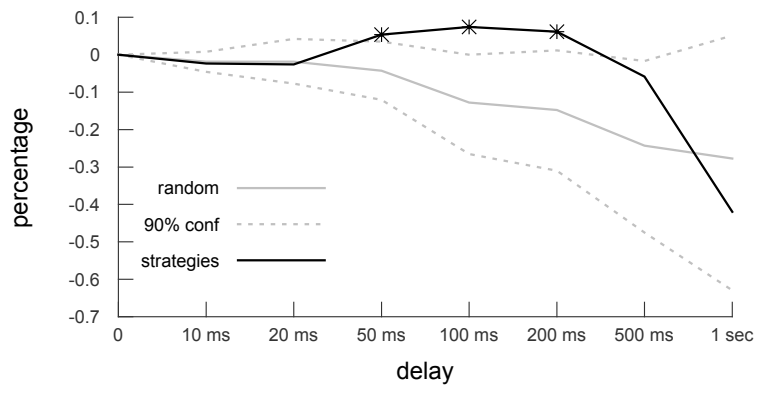

(d) BOTTOM 25\% NEGATIVE

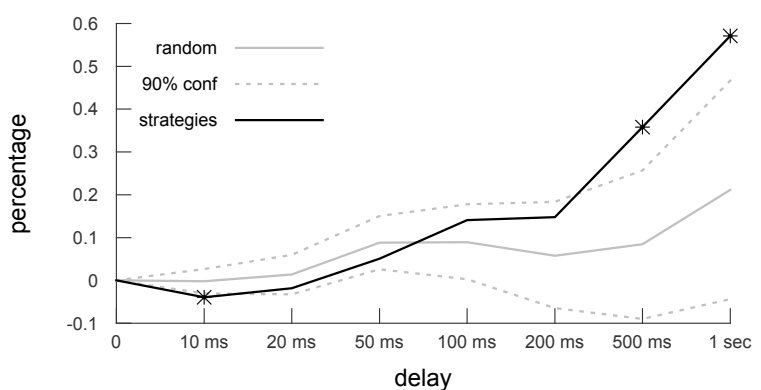


Figure 9: The importance of speed on the days with low volatility (the bottom tercile for the intraday high of the VIX over the period January 6, 2009 to September 30, 2009) for all trading strategies with a positive (a) and negative (c) performance as well as the importance of speed for the top (bottom) quartile of the strategies with a positive (negative) performance in (b) and (d), respectively. All strategies are implemented with a capacity level of 100 ETFs. For different levels of delay (between the moment a trading interval changes and the moment a trade signal is constructed and executed) in milliseconds (ms), the percentage value (corresponding to the solid dark line) indicates how the performance of strategies with instantaneous execution (a delay of $0 \mathrm{~ms}$ ) changes when execution is delayed. The selection bias and corresponding $90 \%$ bootstrapped confidence interval is provided by the solid light line and dashed lines, respectively. In case the importance of speed is significant this is indicated by a $*$.

(a) ALL POSITIVE

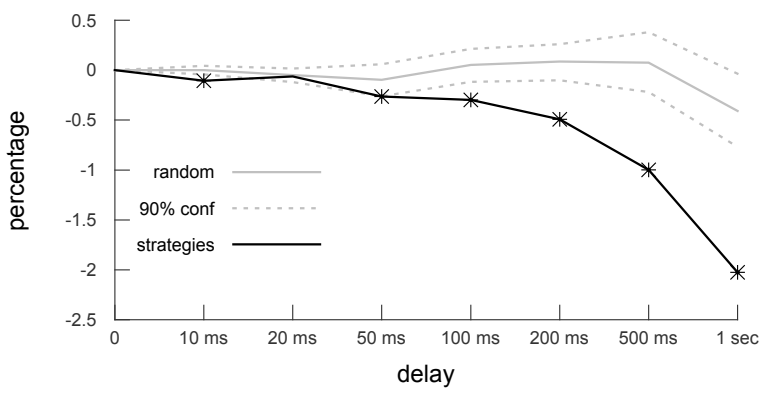

(c) ALL NEGATIVE

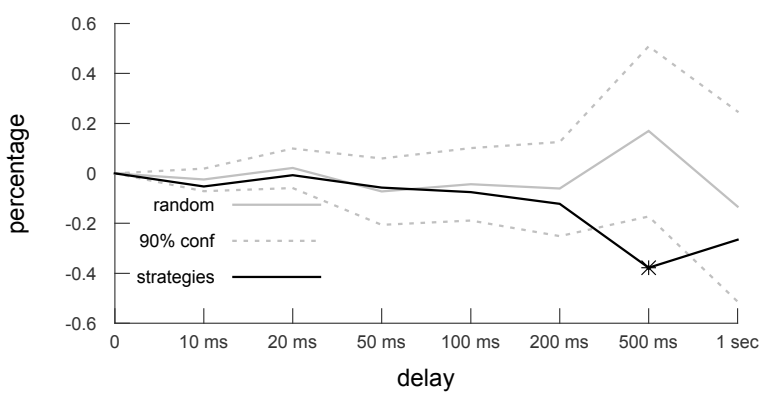

(b) TOP 25\% POSITIVE

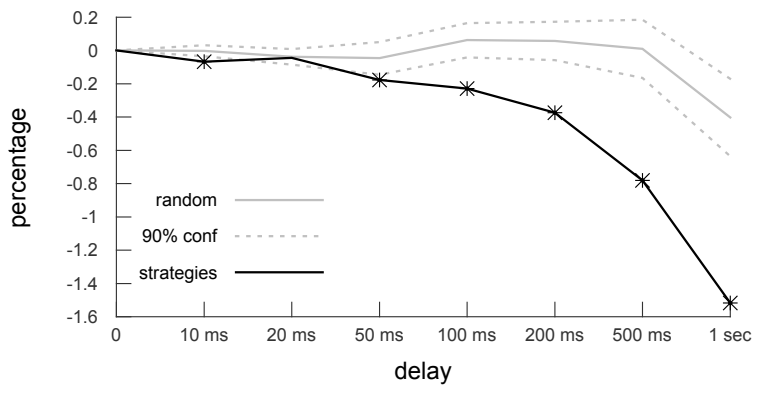

(d) BOTTOM 25\% NEGATIVE

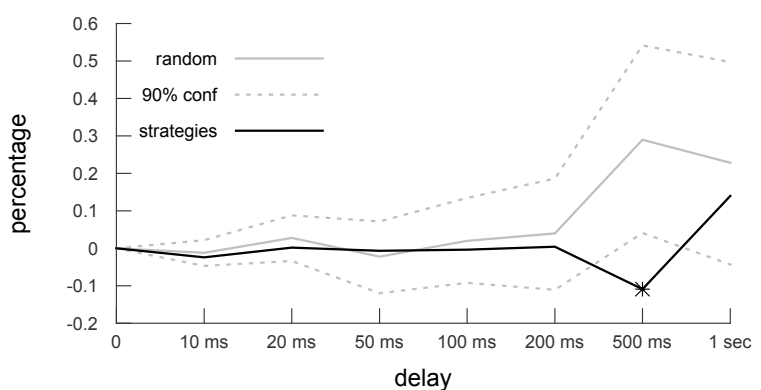



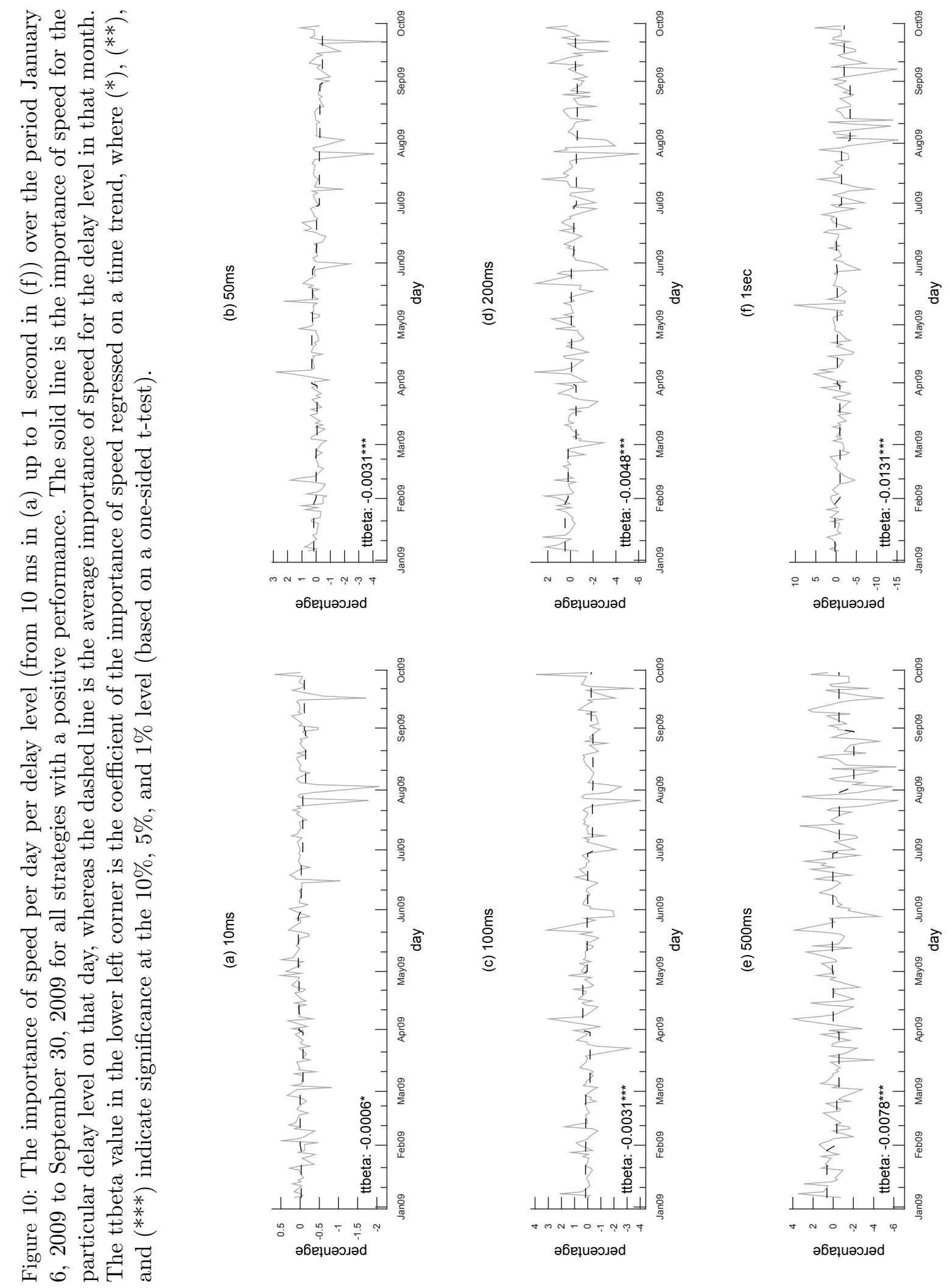
Figure 11: The importance of speed on the days with low volatility (the bottom tercile for the intraday high of the VIX over the period January 6, 2009 to September 30, 2009) for trading strategies with capacity levels of 100, 500, 1,000, and 5,000 ETFs. Subplot (a) and (c) provide the importance of speed for all strategies with a positive (a) and negative (c) performance. In addition, subplots (b) and (d) present the importance of speed for the top (bottom) quartile of the strategies with a positive (negative) performance, respectively. For different levels of delay (between the moment a trading interval changes and the moment a trade signal is constructed and executed) in milliseconds (ms), the percentage value indicates how the performance of strategies with instantaneous execution (a delay of $0 \mathrm{~ms}$ ) changes when execution is delayed. In case the importance of speed is significant this is indicated by a $*$.

(a) ALL POSITIVE

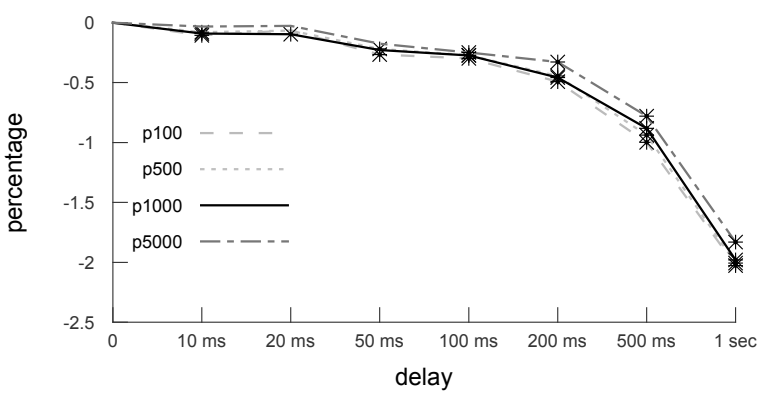

(c) ALL NEGATIVE

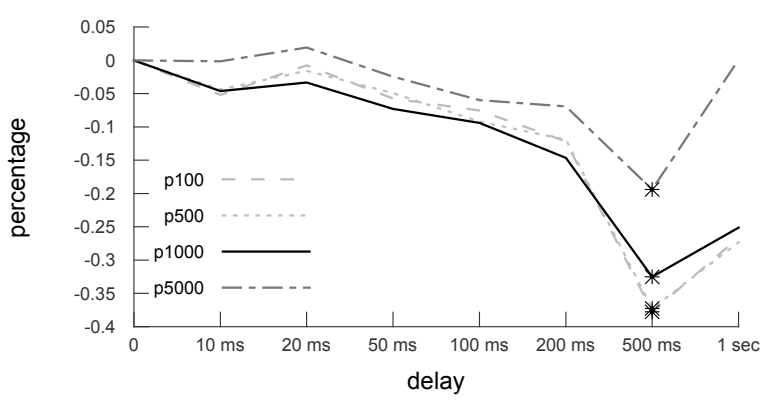

(b) TOP 25\% POSITIVE

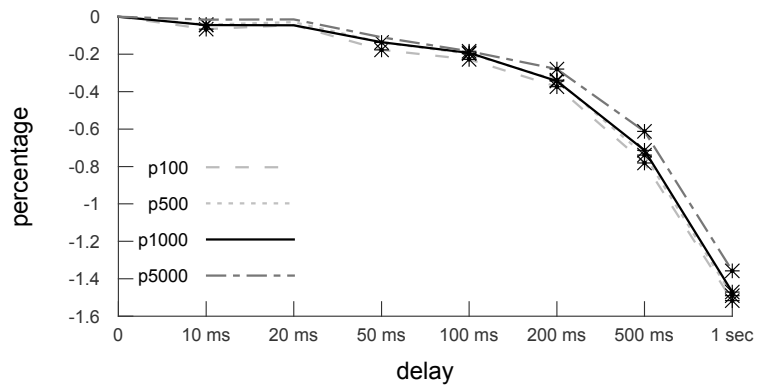

(d) TOP 25\% NEGATIVE

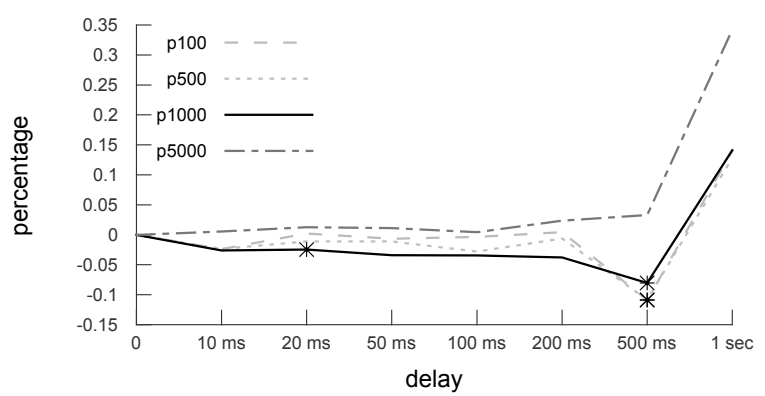




\title{
Appendix A. Rule Settings
}

\author{
Moving Average Settings \\ $30 \mathrm{sec} \quad 10,15,20,25,30,35,40,45,60$ \\ $l: \quad 60 \mathrm{sec} \quad 10,15,20,25,30,35,40,45,60$ \\ $5 \min \quad 4,5,6,7,8,9,10,11,12$ \\ $30 \mathrm{sec} \quad 2,5,10,15,20,25,30$ \\ $s: \quad 60 \mathrm{sec} \quad 2,5,10,15,20,25,30$ \\ 5 min $2,3,4,5,6,7,8$ \\ $30 \mathrm{sec} \quad 0,0.0005,0.001,0.0015,0.0025,0.0035$ \\ $b: \quad 60 \mathrm{sec} \quad 0,0.0005,0.001,0.0015,0.0025,0.004$ \\ $5 \mathrm{~min} \quad 0,0.00025,0.0005,0.00075,0.001,0.0025$ \\ $d: 0,1,2,3$ \\ $h: 1,2,4,6$
}

Support and Resistance Settings

$l: 3,5,10,15,20,25,30,35,40,45,50,55,60$

$30 \mathrm{sec} \quad 0,0.00025, \ldots, 0.001,0.001125,0.00125,0.0015$,

$b: \quad 0.00175,0.002,0.0025$

$60 \mathrm{sec} \quad 0,0.00025,0.0005, \ldots, 0.0025$,

5 min $\quad 0,0.00025, \ldots, 0.001,0.0015, \ldots, 0.0035,0.00375$

$d, h:$ See moving average settings.

\section{Filter Settings}

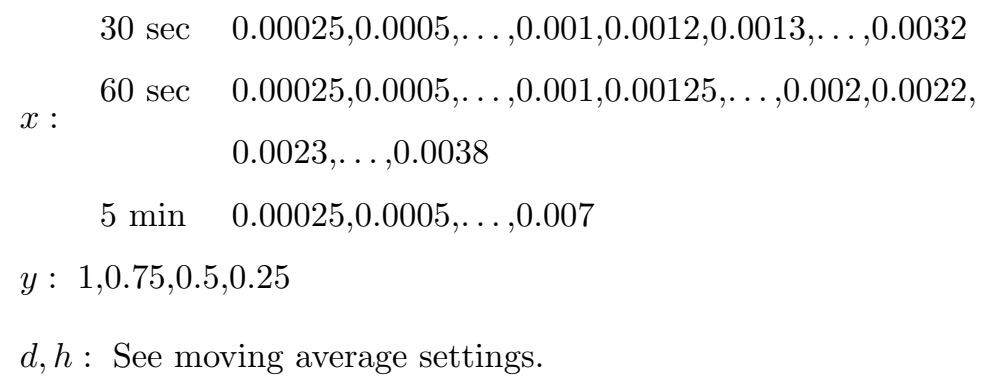




\section{Channel Break-out Settings}

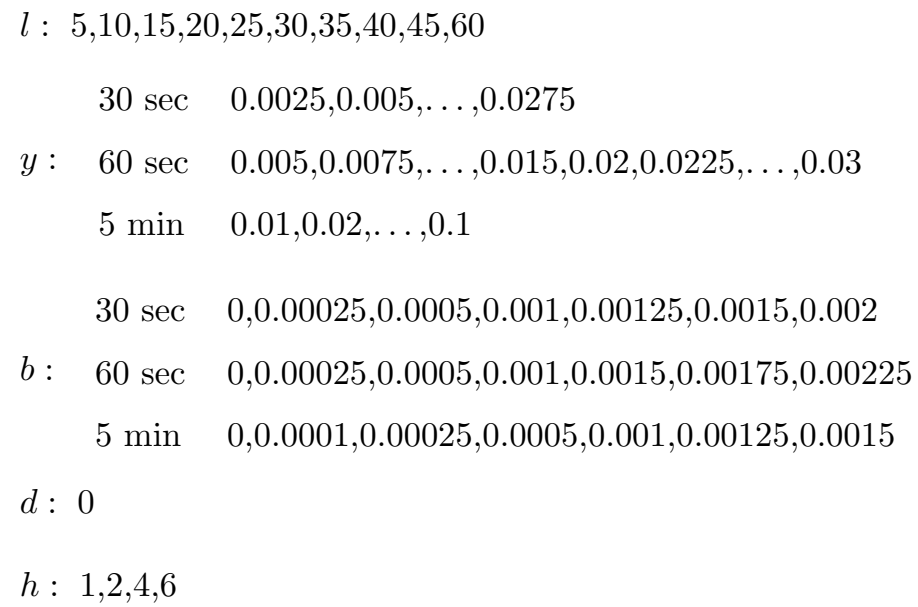

On Balance Volume Settings

$l, s, d, h:$ See the moving average settings.

$30 \mathrm{sec} \quad 0,0.25,0.5,1,1.5,2,3$

$b: \quad 60 \mathrm{sec} \quad 0,0.1,0.25,0.5,1.5,2,3$

5 min $\quad 0,0.05,0.1,0.25,0.5,0.75,1$

Momentum in Price Settings

$l, s, d, h$ : See the moving average settings.

$30 \mathrm{sec} \quad 0,0.00025,0.0005,0.00075,0.001,0.00125,0.0015$

$b: \quad 60 \mathrm{sec} \quad 0,0.00025,0.0005,0.00075,0.001,0.00125,0.00175$

5 min $\quad 0,0.00025,0.0005,0.00075,0.001,0.00125,0.00175$

Momentum in Volume Settings

$l, s, d, h$ : See the moving average settings.

$30 \mathrm{sec} \quad 0,0.25,0.5,1,1.5,2,3.5$

$b: \quad 60 \mathrm{sec} \quad 0,0.1,0.25,0.5,0.75,1.5,2.5$

5 min $\quad 0,0.025,0.05,0.15,0.25,0.5,1$ 


\section{Appendix B. Internet Appendix}

Table B.1: The importance of speed for different delay levels, intervals, and ETFs on all days for a capacity level of 100 ETFs.

\begin{tabular}{|c|c|c|c|c|c|c|c|c|}
\hline & & $10 \mathrm{~ms}$ & $20 \mathrm{~ms}$ & $50 \mathrm{~ms}$ & $100 \mathrm{~ms}$ & $200 \mathrm{~ms}$ & $500 \mathrm{~ms}$ & $1 \mathrm{sec}$ \\
\hline \multirow[t]{3}{*}{ SPY } & $30 \mathrm{sec}$ & -0.0291 & -0.0271 & 0.0383 & 0.0583 & $-0.1587^{*}$ & -0.4025 & -1.0768 \\
\hline & $60 \mathrm{sec}$ & -0.0454 & -0.0457 & -0.0422 & -0.0657 & $-0.2213^{*}$ & $-0.3942^{*}$ & $-1.0481^{*}$ \\
\hline & $5 \mathrm{~min}$ & -0.0254 & -0.0271 & $-0.0095^{*}$ & -0.0673 & -0.2492 & $-0.4834^{*}$ & $-0.9368^{*}$ \\
\hline \multirow[t]{3}{*}{ QQQQ } & $30 \mathrm{sec}$ & -0.0282 & $-0.0165^{*}$ & 0.0279 & -0.0472 & -0.2732 & -0.8791 & $-1.7429 *$ \\
\hline & $60 \mathrm{sec}$ & $-0.0359^{*}$ & $-0.0247^{*}$ & 0.0098 & -0.0729 & -0.2635 & $-1.0090^{*}$ & $-1.9107^{*}$ \\
\hline & $5 \mathrm{~min}$ & -0.0532 & $-0.0543^{*}$ & -0.0682 & -0.1138 & -0.3484 & -0.7286 & $-1.1921^{*}$ \\
\hline \multirow[t]{3}{*}{ IWM } & $30 \mathrm{sec}$ & 0.0021 & $-0.0356^{*}$ & -0.0438 & -0.1558 & -0.3215 & $-1.0056^{*}$ & $-1.7754^{*}$ \\
\hline & $60 \mathrm{sec}$ & 0.0258 & 0.0184 & 0.0125 & -0.0739 & -0.1493 & $-0.6242^{*}$ & $-1.3355^{*}$ \\
\hline & $5 \mathrm{~min}$ & 0.0349 & 0.0255 & -0.0289 & -0.1127 & -0.2686 & $-0.5738^{*}$ & $-1.1187^{*}$ \\
\hline
\end{tabular}

This table provides the importance of speed (in \% with respect to instantaneous execution) for all trading rules with a positive performance on all trading days during the period January 6, 2009 to September 30, 2009 and a capacity level of 100 ETFs. The importance of speed is provided for seven different delay levels (10 ms up to 1 second), three ETFs (SPY, IWM, and QQQQ) and three trading frequencies (30 seconds, 60 seconds, and 5 minutes). For different levels of delay (between the moment a trading interval changes and the moment a signal is implemented) in milliseconds ( $\mathrm{ms}$ ), the percentage value indicates how the performance of strategies with instantaneous execution (a delay of $0 \mathrm{~ms}$ ) changes when execution is delayed. Values with a ${ }^{*}$ are significant. 
Table B.2: The importance of speed for different delay levels, intervals, and ETFs on days with high volatility for a capacity level of 100 ETFs.

\begin{tabular}{ccccccccc}
\hline \multicolumn{1}{c}{ SPY } & & $10 \mathrm{~ms}$ & $20 \mathrm{~ms}$ & $50 \mathrm{~ms}$ & $100 \mathrm{~ms}$ & $200 \mathrm{~ms}$ & $500 \mathrm{~ms}$ & $1 \mathrm{sec}$ \\
& $60 \mathrm{sec}$ & -0.0471 & -0.1017 & $0.0635^{*}$ & $0.1620^{*}$ & 0.1312 & $-0.0303^{*}$ & -0.2973 \\
& $5 \mathrm{~min}$ & -0.0374 & -0.0499 & $0.0909^{*}$ & $0.1152^{*}$ & $0.0904^{*}$ & -0.0425 & -0.4770 \\
\multirow{2}{*}{ QQQQ } & $30 \mathrm{sec}$ & -0.0270 & -0.0433 & $0.0791^{*}$ & $0.0450^{*}$ & $-0.0158^{*}$ & -0.3197 & -0.6122 \\
& $60 \mathrm{sec}$ & 0.0014 & 0.0263 & -0.0996 & $-0.2600^{*}$ & -0.2754 & -0.4114 & -0.7268 \\
& $5 \mathrm{~min}$ & -0.0290 & -0.0364 & -0.1002 & -0.0506 & -0.1929 & -0.2675 & $-0.4914^{*}$ \\
\multirow{2}{*}{ IWM } & & & & & & & & \\
& $30 \mathrm{sec}$ & -0.0155 & $-0.0728^{*}$ & -0.0212 & $-0.0315^{*}$ & $-0.0687^{*}$ & -0.5225 & $-0.9072^{*}$ \\
& $60 \mathrm{sec}$ & -0.0080 & -0.0385 & -0.0189 & $0.0036^{*}$ & -0.0657 & -0.3297 & $-0.6244^{*}$ \\
& $5 \mathrm{~min}$ & $-0.0373^{*}$ & $-0.0303^{*}$ & $-0.1152^{*}$ & -0.1633 & -0.2789 & $-0.5666^{*}$ & $-0.6511^{*}$ \\
\hline
\end{tabular}

This table provides the importance of speed (in \% with respect to instantaneous execution) for all trading rules with a positive performance on high volatility days (the top tercile for the intraday high of the VIX over the period January 6, 2009 to September 30, 2009) and a capacity level of 100 ETFs. The importance of speed is provided for seven different delay levels (10 ms up to 1 second), three ETFs (SPY, IWM, and QQQQ) and three trading frequencies (30 seconds, 60 seconds, and 5 minutes). For different levels of delay (between the moment a trading interval changes and the moment a signal is implemented) in milliseconds (ms), the percentage value indicates how the performance of strategies with instantaneous execution (a delay of $0 \mathrm{~ms}$ ) changes when execution is delayed. Values with a $*$ are significant. 
Figure B.1: Percentage of total message activity over the period January 6, 2009 to June 4, 2009 and September 1, 2009 to September 30, 2009 (the periods during which NASDAQ has no flash order functionality) during normal trading hours due to fleeting orders (a), (b) the distribution of the point of entry of fleeting orders in the orderbook where $0(-1)$ and $x$ indicate additions at the best bid or ask quote, before the best bid or ask quote, and $x$ cents behind the best bid or ask quote (with $1 \leq x \leq 23$ ), respectively. Subplot (c) provides the percentage of all improvements to the best bid or ask quote that dissapear within $10 \mathrm{~ms}$ up to 1 second, whereas (d) shows the percentage of missed opportunities within $10 \mathrm{~ms}$ up to 1 second. A missed opportunity is an add order message which is an improvement to the best bid or ask that, when it is either removed or executed, leaves a worse price in the orderbook.

(a) \% FLEETS OF LIMIT ORDERS

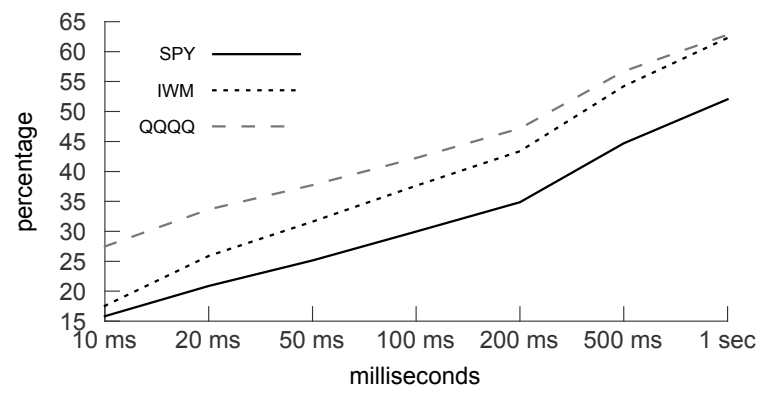

(c) \% OF BBA IMPROVEMENTS THAT DISSAPEAR

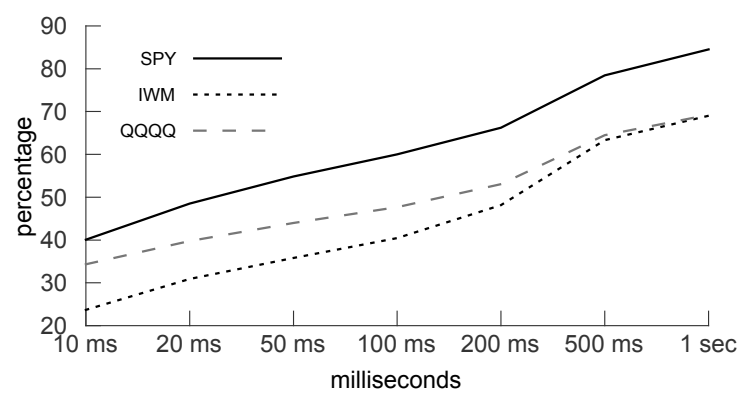

(b) \% FLEETS WRT BBA (50ms)

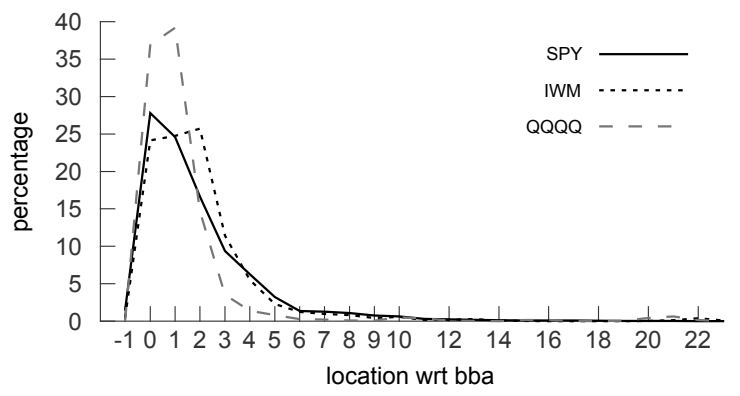

(d) \% OF MISSED OPPORTUNITIES

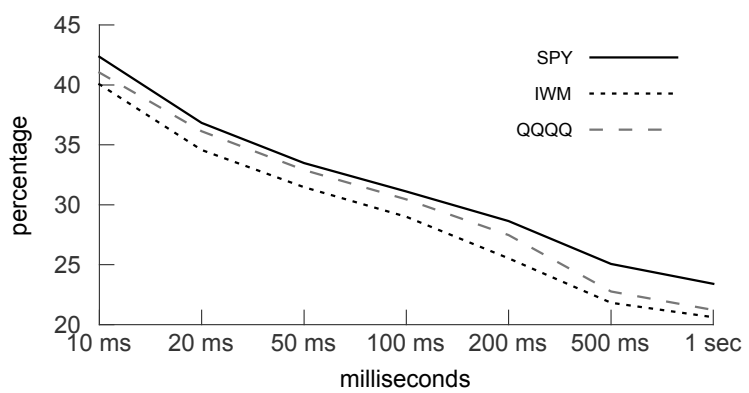


Figure B.2: Percentage of total message activity over the period June 5, 2009 to August 31, 2009 (the period during which NASDAQ has flash order functionality) during normal trading hours due to fleeting orders (a), (b) the distribution of the point of entry of fleeting orders in the orderbook where 0 $(-1)$ and $x$ indicate additions at the best bid or ask quote, before the best bid or ask quote, and $x$ cents behind the best bid or ask quote (with $1 \leq x \leq 23$ ), respectively. Subplot (c) provides the percentage of all improvements to the best bid or ask quote that dissapear within $10 \mathrm{~ms}$ up to 1 second, whereas (d) shows the percentage of missed opportunities within $10 \mathrm{~ms}$ up to 1 second. A missed opportunity is an add order message which is an improvement to the best bid or ask that, when it is either removed or executed, leaves a worse price in the orderbook.

(a) \% FLEETS OF LIMIT ORDERS

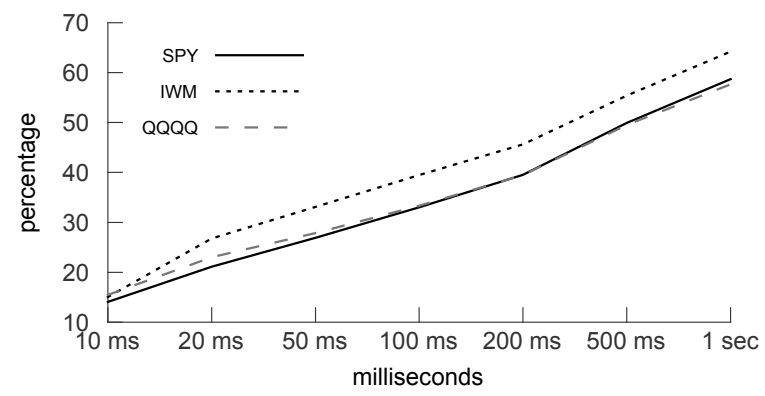

(c) \% OF BBA IMPROVEMENTS THAT DISSAPEAR

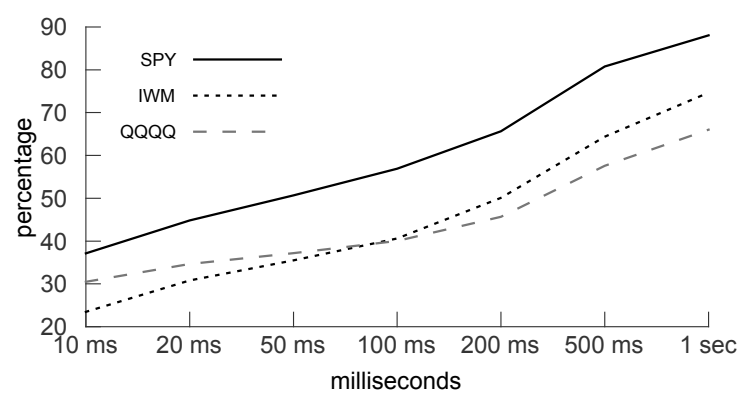

(b) \% FLEETS WRT BBA (50ms)

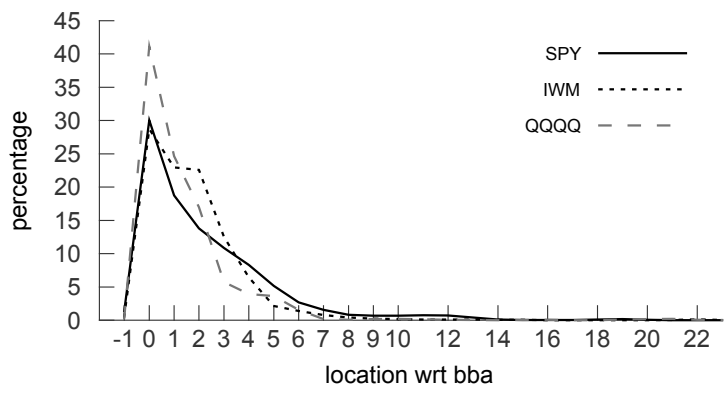

(d) \% OF MISSED OPPORTUNITIES

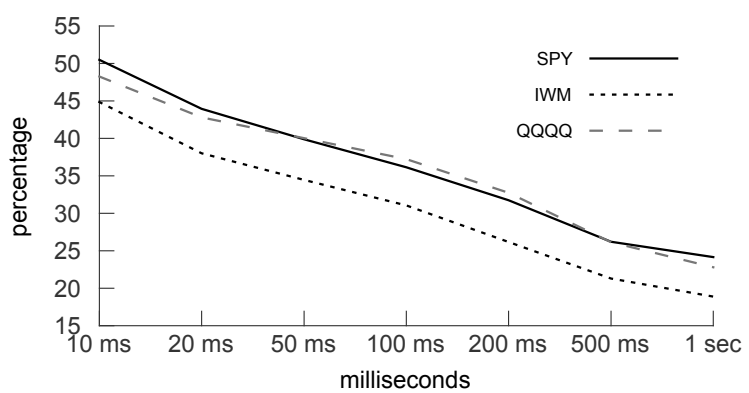


Figure B.3: The relative importance of speed per rule per day on the days with low volatility (the bottom tercile for the intraday high of the VIX over the period January 6, 2009 to September 30, 2009) for all trading strategies with a positive (a) and negative (c) performance as well as the importance of speed for the top (bottom) quartile of the strategies with a positive (negative) performance in (b) and (d), respectively. The importance of speed is calculated for each individual trading rule (by calculating the relative difference of the performance with a delay and the performance resulting from instantaneous execution) per day and is then averaged over all trading rules per day and all days in the sample period. All strategies are implemented with a capacity level of 100 ETFs. For different levels of delay (between the moment a trading interval changes and the moment a trade signal is constructed and executed) in milliseconds (ms), the percentage value (corresponding to the solid dark line) indicates how the performance of strategies with instantaneous execution (a delay of $0 \mathrm{~ms}$ ) changes when execution is delayed. The selection bias and corresponding $90 \%$ bootstrapped confidence interval is provided by the solid light line and dashed lines, respectively. In case the importance of speed is significant this is indicated by a $*$.

(a) ALL POSITIVE

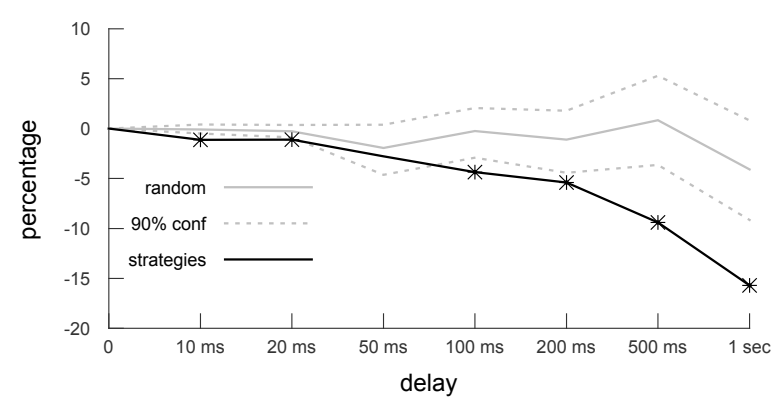

(c) ALL NEGATIVE

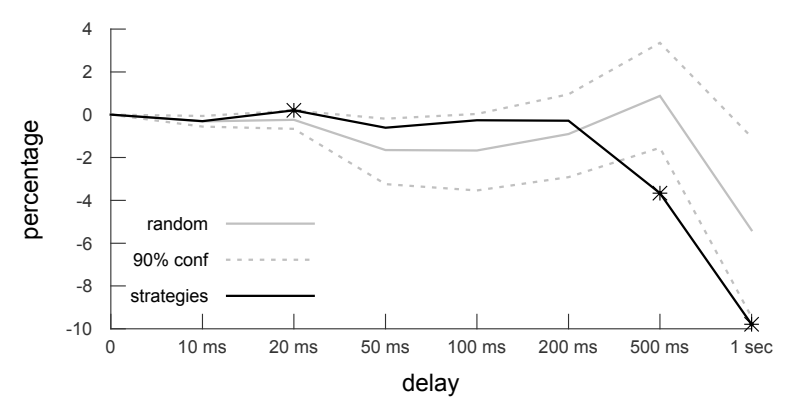

(b) TOP $25 \%$ POSITIVE

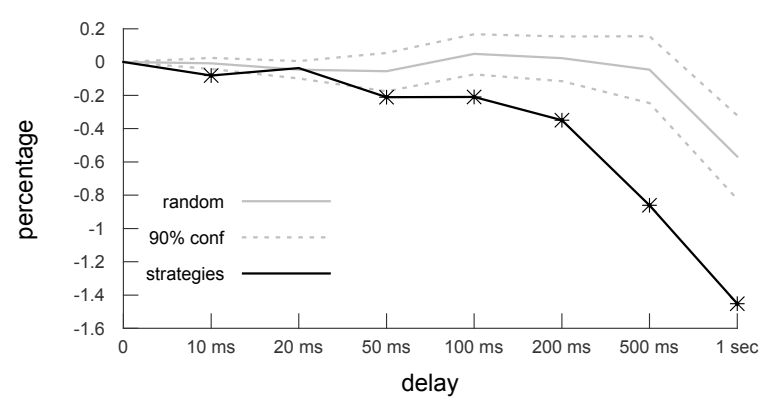

(d) BOTTOM $25 \%$ NEGATIVE

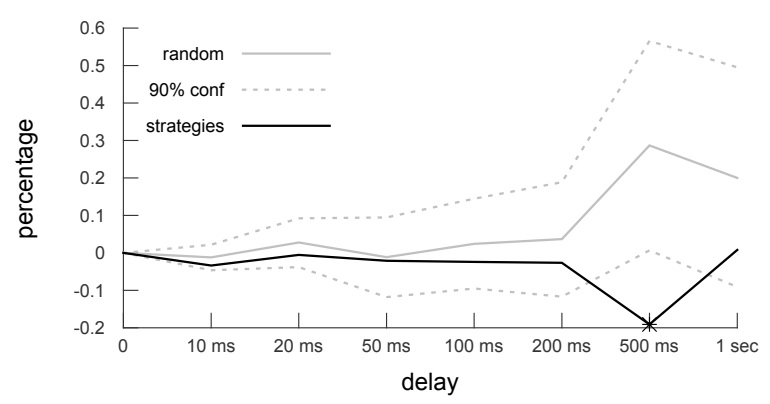


Figure B.4: The absolute importance of speed per day (which, for absolute differences, is identical to the absolute importance of speed per rule per day) in annualized, absolute basispoints (bps) on the days with low volatility (the bottom tercile for the intraday high of the VIX over the period January 6, 2009 to September 30, 2009) for all trading strategies with a positive (a) and negative (c) performance as well as the importance of speed for the top (bottom) quartile of the strategies with a positive (negative) performance in (b) and (d), respectively. The importance of speed is the absolute difference of the average trading rule return per day for strategies with a delay of $10 \mathrm{~ms}$ up to 1 second with the average trading rule return per day for strategies with instantaneous execution. Annualization is done by multiplying the average basispoint difference with 252 trading days per year. All strategies are implemented with a capacity level of 100 ETFs. For different levels of delay (between the moment a trading interval changes and the moment a trade signal is constructed and executed) in milliseconds (ms), the basispoints (bps) value (corresponding to the solid dark line) indicates how the performance of strategies with instantaneous execution (a delay of $0 \mathrm{~ms}$ ) changes when execution is delayed. The selection bias and corresponding $90 \%$ bootstrapped confidence interval is provided by the solid light line and dashed lines, respectively. In case the importance of speed is significant this is indicated by a $*$.

(a) ALL POSITIVE

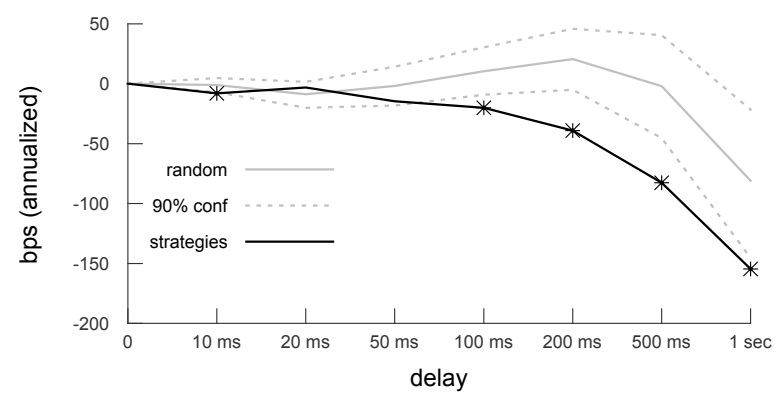

(c) ALL NEGATIVE

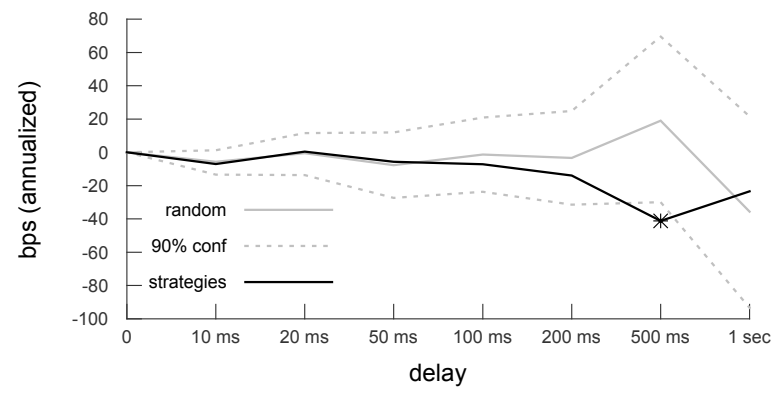

(b) TOP 25\% POSITIVE

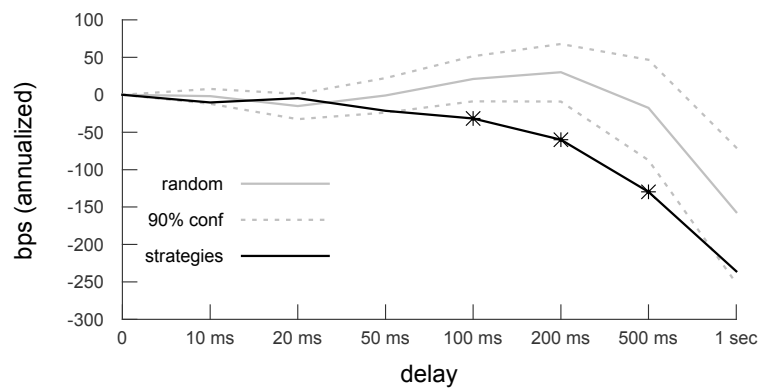

(d) BOTTOM 25\% NEGATIVE

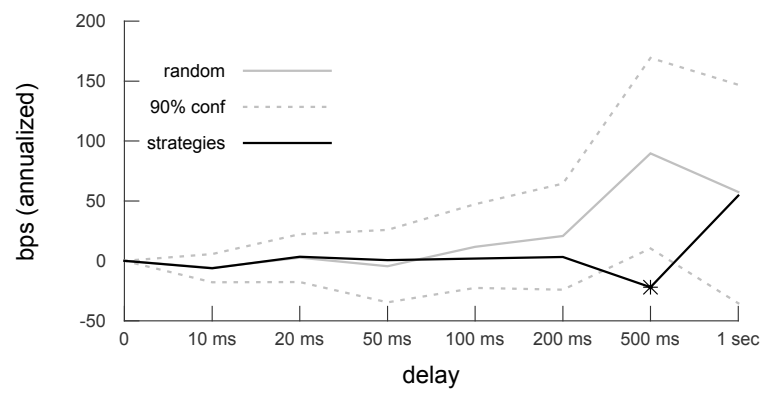


Figure B.5: The relative truncated importance of speed per rule per day on the days with low volatility (the bottom tercile for the intraday high of the VIX over the period January 6, 2009 to September 30, 2009) for all trading strategies with a positive (a) and negative (c) performance as well as the importance of speed for the top (bottom) quartile of the strategies with a positive (negative) performance in (b) and (d), respectively. All strategies are implemented with a capacity level of 100 ETFs. The importance of speed is calculated for each individual trading rule (by calculating the relative difference of the performance with a delay and the performance resulting from instantaneous execution) per day and is then averaged over all trading rules per day and all days in the sample period. Individual relative differences larger (smaller) than 100\% (-100\%) are truncated to $100 \%$ and $-100 \%$, respectively. For different levels of delay (between the moment a trading interval changes and the moment a trade signal is constructed and executed) in milliseconds (ms), the percentage value (corresponding to the solid dark line) indicates how the performance of strategies with instantaneous execution (a delay of $0 \mathrm{~ms}$ ) changes when execution is delayed. The selection bias and corresponding $90 \%$ bootstrapped confidence interval is provided by the solid light line and dashed lines, respectively. In case the importance of speed is significant this is indicated by a $*$.

(a) ALL POSITIVE

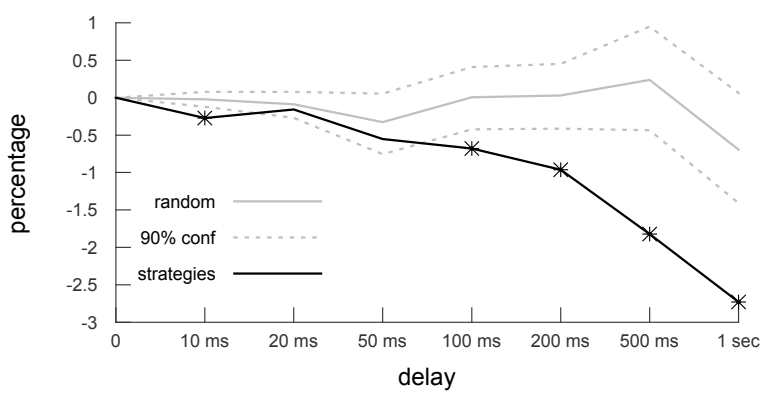

(c) ALL NEGATIVE

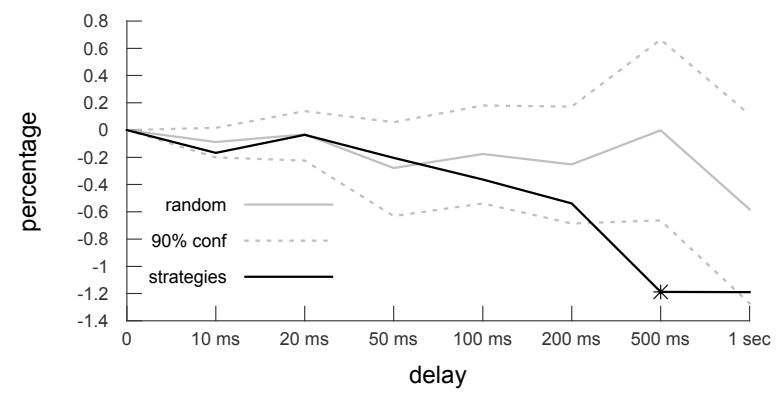

(b) TOP $25 \%$ POSITIVE

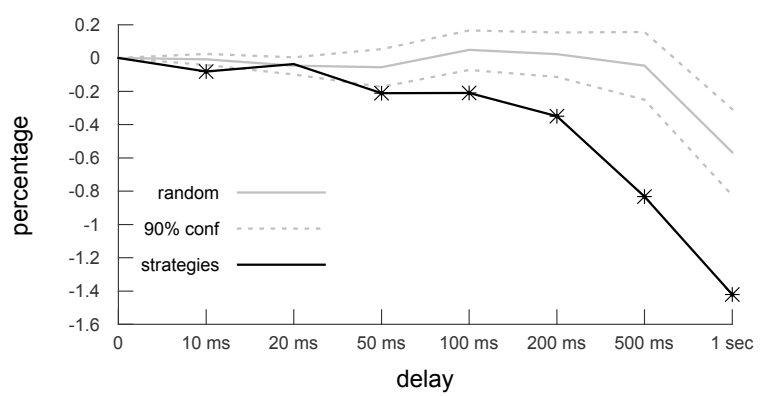

(d) BOTTOM $25 \%$ NEGATIVE

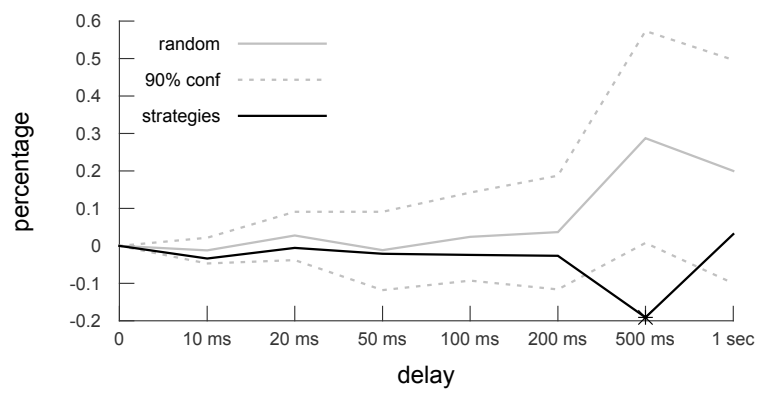


Figure B.6: The nonparametric importance of speed on the days with low volatility (the bottom tercile for the intraday high of the VIX over the period January 6, 2009 to September 30, 2009) for all trading strategies with a positive (a) and negative (c) performance as well as the importance of speed for the top (bottom) quartile of the strategies with a positive (negative) performance in (b) and (d), respectively. All strategies are implemented with a capacity level of 100 ETFs. For different levels of delay (between the moment a trading interval changes and the moment a trade signal is constructed and executed) in milliseconds (ms), the percentage value (corresponding to the solid dark line) indicates how frequencly the performance of strategies with a delay decreases (solid dark line), remains the same (light grey line), and increases (dashed dark line). A (two-sided) Wilcoxon signed-rank test is performed to check whether the percentage of decreases is significantly different from the percentage of increases due to the introduction of a delay in execution. Significant values at the $10 \%, 5 \%$, and $1 \%$ level are indicated by a $\odot, \bullet$, and $*$, respectively.

(a) ALL POSITIVE

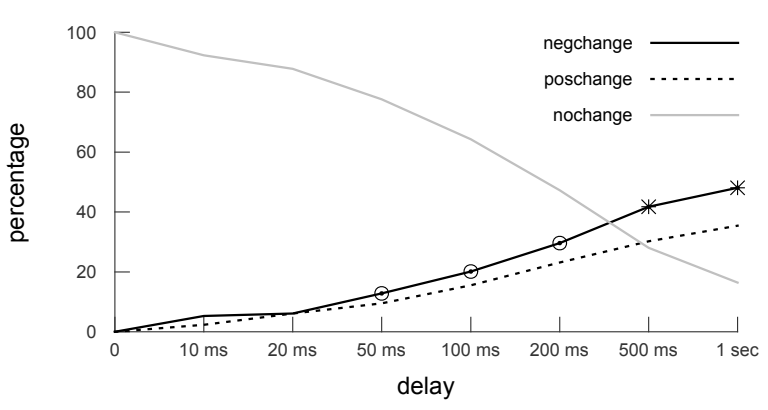

(c) ALL NEGATIVE

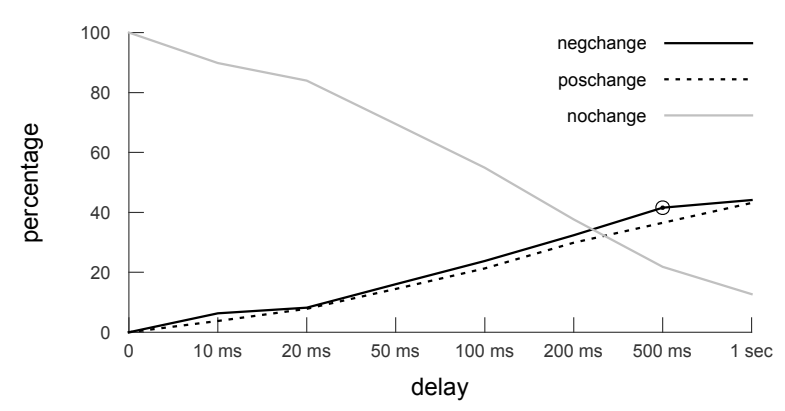

(b) TOP 25\% POSITIVE

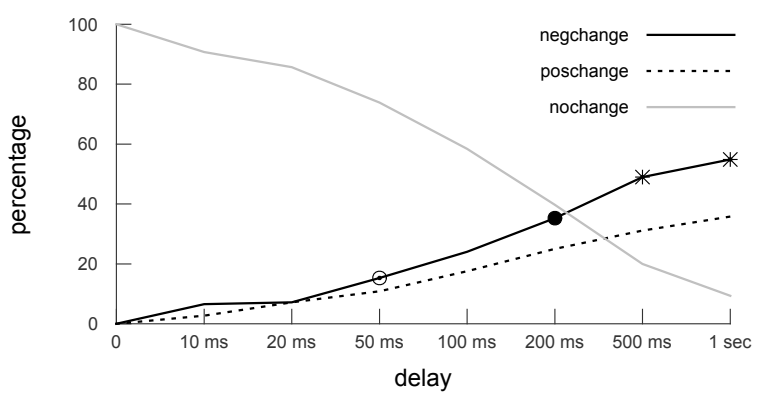

(d) BOTTOM $25 \%$ NEGATIVE

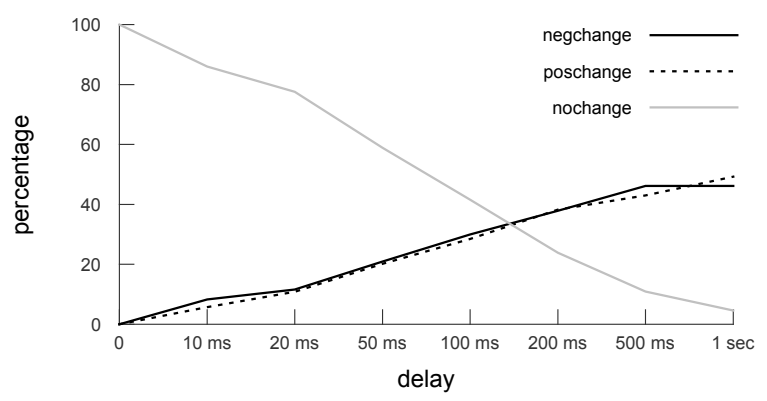

